\title{
CHARACTERS OF FEIGIN-STOYANOVSKY'S TYPE SUBSPACES OF LEVEL ONE MODULES FOR AFFINE LIE ALGEBRAS OF TYPES $A_{\ell}^{(1)}$ AND $D_{4}^{(1)}$
}

\author{
Goran TrupČEviĆ
}

University of Zagreb, Croatia

\begin{abstract}
We use combinatorial description of bases of FeiginStoyanovsky's type subspaces of standard modules of level 1 for affine Lie algebras of types $A_{\ell}^{(1)}$ and $D_{4}^{(1)}$ to obtain character formulas. These descriptions naturally lead to systems of recurrence relations for which we also find solutions.
\end{abstract}

\section{INTRODUCTION}

Principal subspaces were introduced by B. L. Feigin and A. Stoyanovsky in [13] where they gave a construction of bases of standard modules $L(\Lambda)$ consisting of semi-infinite monomials and monomial bases of their principal subspaces, and also calculated characters of both principal subspaces and the whole standard modules for affine Lie algebra $\tilde{\mathfrak{g}}$ of type $A_{1}^{(1)}$. A similar approach was used by M. Primc in $[19,20]$ where he constructed semi-infinite monomial bases for all standard modules for affine Lie algebras of type $A_{\ell}^{(1)}$ and for basic modules $L\left(\Lambda_{0}\right)$ for any classical affine Lie algebra. Instead of principal subspaces of Feigin and Stoyanovsky, Primc used so-called FeiginStoyanovsky's type subspace. Later, in [10] it was noted that bases of FeiginStoyanovsky's type subspaces from [19] were parameterized by $(k, \ell+1)$ admissible configurations which were studied in [10-12].

G. Georgiev generalized Feigin-Stoyanovsky's results to a certain class of standard modules for affine Lie algebras of type $A_{\ell}^{(1)}$ (see [14]). In the proof of

2010 Mathematics Subject Classification. 17B67, 05A19.

Key words and phrases. Affine Lie algebras, principal subspaces, character formulas.

Partially supported by the Ministry of Science and Technology of the Republic of Croatia, Project ID 037-0372794-2806. 
linear independence, Georgiev used intertwining operators between standard modules. S. Capparelli, J. Lepowsky and A. Milas in $[8,9]$ used intertwining operators to obtain exact sequences of principal subspaces and recurrence relations for their characters. This approach was further investigated in [3-7].

Motivated by Georgiev's and Capparelli-Lepowsky-Milas' way of using intertwining operators, Primc gave in [21] a simpler proof of linear independence of bases from [19], and in $[1,2,22,23]$ new constructions of bases in $A_{\ell}^{(1)}$ and $D_{\ell}^{(1)}$ cases were given. Furthermore, M. Jerković in [15] used the proof of linear independence from [21] to obtain exact sequences of FeiginStoyanovsky's type subspaces and recurrence relations for the corresponding characters in the $A_{\ell}^{(1)}$-case. By solving these relations, Jerković in [16] obtained character formulas in the $A_{2}^{(1)}$-case, which agreed with formulas from $[11,12]$.

In this paper we use combinatorial description of bases of Feigin-Stoyanovsky's type subspaces of standard modules of level 1 from $[1,20,23]$ to obtain character formulas. These descriptions naturally lead to systems of recurrence relations for which we also find solutions.

Let $\mathfrak{g}$ be a simple complex Lie algebra, $\mathfrak{h} \subset \mathfrak{g}$ its Cartan subalgebra, $R$ the corresponding root system. Let $\mathfrak{g}=\mathfrak{h}+\sum_{\alpha \in R} \mathfrak{g}_{\alpha}$ be a root decomposition of $\mathfrak{g}$. Fix root vectors $x_{\alpha} \in \mathfrak{g}_{\alpha}$. Let $\langle\cdot, \cdot\rangle$ be a normalized invariant bilinear form on $\mathfrak{g}$, and by the same symbol denote the induced form on $\mathfrak{g}^{*}$. Let $\Pi=\left\{\alpha_{1}, \ldots, \alpha_{\ell}\right\}$ be a basis of the root system $R$, and let $\left\{\omega_{1}, \ldots, \omega_{\ell}\right\}$ be the corresponding set of fundamental weights. Fix a minuscule fundamental weight $\omega$ and set $\Gamma=\{\gamma \in R \mid\langle\gamma, \omega\rangle=1\}, \mathfrak{g}_{1}=\sum_{\alpha \in \Gamma} \mathfrak{g}_{\alpha}$. The set $\Gamma$ is called the set of colors.

Let $\tilde{\mathfrak{g}}=\mathfrak{g} \otimes \mathbb{C}\left[t, t^{-1}\right] \oplus \mathbb{C} c \oplus \mathbb{C} d$ be the associated affine Lie algebra, where $c$ is the canonical central element, and $d$ is the degree operator. Elements $x_{\alpha}(r)=x_{\alpha} \otimes t^{r}$ are fixed real root vectors. Let $\tilde{\mathfrak{g}}_{1}=\mathfrak{g}_{1} \otimes \mathbb{C}\left[t, t^{-1}\right]$, a commutative Lie subalgebra with a basis $\left\{x_{\gamma}(-r) \mid r \in \mathbb{Z}, \gamma \in \Gamma\right\}$. Let $L(\Lambda)$ be a standard $\tilde{\mathfrak{g}}$-module of level 1 , with a fixed highest weight vector $v_{\Lambda}$. A FeiginStoyanovsky's type subspace of $L(\Lambda)$ is a $\tilde{\mathfrak{g}}_{1}$-submodule of $L(\Lambda)$ generated with $v_{\Lambda}$

$$
W(\Lambda)=U\left(\tilde{\mathfrak{g}}_{1}\right) \cdot v_{\Lambda} \subset L(\Lambda)
$$

For the Lie algebra $\mathfrak{g}$ of type $A_{\ell}$ it was shown in $[20,22]$ that monomial vectors $\underline{x} v_{\Lambda}$, where $\underline{x}=x_{\gamma_{n}}\left(-r_{n}\right) \cdots x_{\gamma_{1}}\left(-r_{1}\right), \gamma_{i} \in \Gamma, r_{i} \in \mathbb{N}$, such that $\underline{x}$ satisfy certain combinatorial conditions called difference and initial conditions, constitute a basis of $W(\Lambda)$. The analogous fact was proved in $[1,20]$ for $\mathfrak{g}$ of type $D_{\ell}$.

To obtain character formula when Lie algebra $\mathfrak{g}$ is of type $A_{\ell}$, we first consider two particular cases, when $\omega=\omega_{1}$ and $\omega=\omega_{\ell}$; these are the cases that were considered in $[10-12,15-17,19]$, but also for higher-level modules. For every $\mathfrak{h}$-weight subspace of $W(\Lambda)$, we construct a bijection between the 
basis of that subspace and products of partitions of certain length. This gives formulas (3.7) and (3.8) for the character of $W(\Lambda)$, that were already known (e.g. in [17]). In the case $\omega=\omega_{m}, 1<m<\ell$, the set of colors $\Gamma$ can be decomposed into a product of "rows" and "columns". The sets of rows and columns can be regarded as sets of colors for the two particular cases that have already been considered. For a given $\mathfrak{h}$-weight subspace of $W(\Lambda)$, we consider its basis elements $\underline{x} v_{\Lambda}$, where $\underline{x}=x_{\gamma_{n}}\left(-r_{n}\right) \cdots x_{\gamma_{1}}\left(-r_{1}\right), \gamma_{i} \in \Gamma, r_{i} \in \mathbb{N}$. To every such basis element we can attach its path $\mathbf{p}(\underline{x})=\left(\gamma_{n}, \ldots, \gamma_{1}\right)$, and conversely, to every path $\mathbf{p}$ we can attach a basis element $\underline{x}(\mathbf{p})$ that will be minimal in some sense. By the decomposition of $\Gamma$, for every path $\mathbf{p}$ in $\Gamma$, we have the corresponding paths of rows and columns. We use character formulas for the two particular cases to find "graded cardinality" of the set of "minimal" monomials for paths corresponding to the given $\mathfrak{h}$-weight. From this we obtain formula (3.21) for the character of $W(\Lambda)$.

When Lie algebra $\mathfrak{g}$ is of type $D_{4}$, we decompose the set of colors into two subsets that correspond to the cases $A_{2}$, with $\omega=\omega_{2}$, and $A_{3}$, with $\omega=\omega_{2}$. We use character formulas (3.7) and (3.21) for the latter cases to obtain character formula $(4.35)$ in the $D_{4}$-case.

Both in $A_{\ell}$ and $D_{4}$ cases, descriptions of combinatorial bases naturally lead to systems of recurrence relations. We can find solutions of these systems in a similar way to the one we used for calculating character formulas of FeiginStoyanovsky's type subspaces.

The outline of this paper is as follows: in Section 2 we introduce basic definitions. In Section 3 we find character formulas in the $A_{\ell \text {-case. We also }}$ find solutions of the corresponding system of recurrence relations. In Section 4 we do the same thing in the $D_{4}$-case.

\section{Feigin-Stoyanovsky's type SUBSPACE}

Let $\mathfrak{g}$ be a simple finite-dimensional Lie algebra. Let $\mathfrak{h} \subset \mathfrak{g}$ be a Cartan subalgebra of $\mathfrak{g}$ and $R$ the corresponding root system. Fix a basis $\Pi=\left\{\alpha_{1}, \ldots, \alpha_{\ell}\right\}$ of $R$. Then we have the root decomposition $\mathfrak{g}=\mathfrak{h} \oplus \coprod_{\alpha \in R} \mathfrak{g}_{\alpha}$ and the triangular decomposition $\mathfrak{g}=\mathfrak{n}_{-} \oplus \mathfrak{h} \oplus \mathfrak{n}_{+}$. Let $\theta=k_{1} \alpha_{1}+\cdots+k_{\ell} \alpha_{\ell}$ be the maximal root. Let $\langle\cdot, \cdot\rangle$ be a a normalized invariant bilinear form on $\mathfrak{g}$ such that $\langle\theta, \theta\rangle=2$; we identify $\mathfrak{h}$ with $\mathfrak{h}^{*}$ via $\langle\cdot, \cdot\rangle$. For $\alpha \in R$ let $\alpha^{\vee}=2 \alpha /\langle\alpha, \alpha\rangle$ denote the corresponding coroot. Also for each root $\alpha \in R$ fix a root vector $x_{\alpha} \in \mathfrak{g}_{\alpha}$. Let $\left\{\omega_{1}, \ldots, \omega_{\ell}\right\}$ be the set of fundamental weights of $\mathfrak{g},\left\langle\omega_{i}, \alpha_{j}\right\rangle=\delta_{i j}, i, j=1, \ldots, \ell$. Denote by $Q=\sum_{i=1}^{\ell} \mathbb{Z} \alpha_{i}$ the root lattice, and by $P=\sum_{i=1}^{\ell} \mathbb{Z} \omega_{i}$ the weight lattice of $\mathfrak{g}$. Denote by $P^{+}=\sum_{i=1}^{\ell} \mathbb{Z}_{\geq 0} \omega_{i}$ the set of dominant integral weights.

Let $\tilde{\mathfrak{g}}$ be the associated untwisted affine Lie algebra,

$$
\tilde{\mathfrak{g}}=\mathfrak{g} \otimes \mathbb{C}\left[t, t^{-1}\right] \oplus \mathbb{C} c \oplus \mathbb{C} d,
$$


with commutation relations

$$
\begin{aligned}
& {[x(i), y(j)]=[x, y](i+j)+i\langle x, y\rangle \delta_{i+j, 0} c,} \\
& {[c, \tilde{\mathfrak{g}}]=0, \quad[d, x(j)]=j x(j),}
\end{aligned}
$$

where $x(j)=x \otimes t^{j}$ for $x \in \mathfrak{g}, j \in \mathbb{Z}$ (cf. [18]).

Set $\mathfrak{h}^{e}=\mathfrak{h} \oplus \mathbb{C} c \oplus \mathbb{C} d, \tilde{\mathfrak{n}}_{ \pm}=\mathfrak{g} \otimes t^{ \pm 1} \mathbb{C}\left[t^{ \pm 1}\right] \oplus \mathfrak{n}_{ \pm}$. Then $\tilde{\mathfrak{g}}$ also has the triangular decomposition $\tilde{\mathfrak{g}}=\tilde{\mathfrak{n}}_{-} \oplus \mathfrak{h}^{e} \oplus \tilde{\mathfrak{n}}_{+}$. Usual extensions of bilinear forms $\langle\cdot, \cdot\rangle$ onto $\mathfrak{h}^{e}$ and $\left(\mathfrak{h}^{e}\right)^{*}$ are denoted by the same symbols (we take $\langle c, d\rangle=1$ ). Denote by $\alpha_{0}, \alpha_{1}, \ldots, \alpha_{\ell} \in\left(\mathfrak{h}^{e}\right)^{*}$ the simple roots, and by $\Lambda_{0}, \Lambda_{1}, \ldots, \Lambda_{\ell} \in$ $\left(\mathfrak{h}^{e}\right)^{*}$ the corresponding fundamental weights. Then $\Lambda_{0}(c)=1, \Lambda_{i}(c)=k_{i}$ for $i=1, \ldots, \ell$.

Weight $\omega \in P$ is said to be minuscule if $\langle\omega, \alpha\rangle \in\{-1,0,1\}$ for $\alpha \in R$. A dominant integral weight $\omega \in P^{+}$is minuscule if and only if $\langle\omega, \theta\rangle=1$.

Fix a minuscule weight $\omega \in P$. Set

$$
\Gamma=\{\alpha \in R \mid\langle\alpha, \omega\rangle=1\} .
$$

Then

$$
\mathfrak{g}=\mathfrak{g}_{-1} \oplus \mathfrak{g}_{0} \oplus \mathfrak{g}_{1}
$$

where

$$
\mathfrak{g}_{0}=\mathfrak{h} \oplus \sum_{\langle\alpha, \omega\rangle=0} \mathfrak{g}_{\alpha}, \quad \mathfrak{g}_{ \pm 1}=\sum_{\alpha \in \pm \Gamma} \mathfrak{g}_{\alpha}
$$

is a $\mathbb{Z}$-gradation of $\mathfrak{g}$. Subalgebras $\mathfrak{g}_{1}$ and $\mathfrak{g}_{-1}$ are commutative. We call elements $\gamma \in \Gamma$ colors and the set $\Gamma$ the set of colors.

The $\mathbb{Z}$-gradation of $\mathfrak{g}$ induces the $\mathbb{Z}$-gradation of affine Lie algebra $\tilde{\mathfrak{g}}$ :

$$
\begin{gathered}
\tilde{\mathfrak{g}}=\tilde{\mathfrak{g}}_{-1}+\tilde{\mathfrak{g}}_{0}+\tilde{\mathfrak{g}}_{1}, \\
\tilde{\mathfrak{g}}_{0}=\mathfrak{g}_{0} \otimes \mathbb{C}\left[t, t^{-1}\right] \oplus \mathbb{C} c \oplus \mathbb{C} d, \quad \tilde{\mathfrak{g}}_{ \pm 1}=\mathfrak{g}_{ \pm 1} \otimes \mathbb{C}\left[t, t^{-1}\right] .
\end{gathered}
$$

Again, $\tilde{\mathfrak{g}}_{-1}$ and $\tilde{\mathfrak{g}}_{1}$ are commutative subalgebras. Set $\tilde{\mathfrak{g}}_{1}^{-}=\tilde{\mathfrak{g}}_{1} \cap \tilde{\mathfrak{n}}_{-}$.

Let $L\left(\Lambda_{k}\right)$ be a standard (i.e., integrable highest weight) $\tilde{\mathfrak{g}}$-module of level $\Lambda_{k}(c)=1$. Denote by $v_{\Lambda_{k}}$ the highest weight vector of $L\left(\Lambda_{k}\right)$. Define a Feigin-Stoyanovsky's type subspace

$$
W\left(\Lambda_{k}\right)=U\left(\tilde{\mathfrak{g}}_{1}\right) \cdot v_{\Lambda_{k}}=U\left(\tilde{\mathfrak{g}}_{1}^{-}\right) \cdot v_{\Lambda_{k}} \subset L\left(\Lambda_{k}\right) .
$$

Set

$$
\tilde{\Gamma}=\left\{x_{\gamma}(-r) \mid \gamma \in \Gamma, r \in \mathbb{Z}\right\}, \quad \tilde{\Gamma}^{-}=\left\{x_{\gamma}(-r) \mid \gamma \in \Gamma, r \in \mathbb{N}\right\}
$$

Since the subalgebra $\tilde{\mathfrak{g}}_{1}$ is commutative, we have $U\left(\tilde{\mathfrak{g}}_{1}\right) \cong \mathbb{C}[\tilde{\Gamma}]$ and $U\left(\tilde{\mathfrak{g}}_{1}^{-}\right) \cong$ $\mathbb{C}\left[\tilde{\Gamma}^{-}\right]$. We often refer to elements of $\tilde{\Gamma}$ as to variables, elements or factors of a monomial from $U\left(\tilde{\mathfrak{g}}_{1}\right)$. 


\section{The CASe $A_{\ell}, \ell \geq 1$}

Let $\mathfrak{g}$ be a simple finite-dimensional Lie algebra of type $A_{\ell}$. In this case all fundamental weights are minuscule. Fix a minuscule weight $\omega=\omega_{m}$, $m \in\{1, \ldots, \ell\}$. The set of colors $\Gamma$ is parameterized by two sets of indices

$$
\Gamma=\{(i j) \mid i=1, \ldots, m ; j=m, \ldots, \ell\},
$$

where

$$
(i j)=\alpha_{i}+\cdots+\alpha_{m}+\cdots+\alpha_{j},
$$

and thus we can think of it as a rectangle with rows ranging from 1 to $m$, and columns ranging from $m$ to $\ell$ (see Figure 1 in [22]). By $x_{i j} \in \mathfrak{g}$ we denote the fixed root vector corresponding to the color $(i j)$.

Linear order $<$ on the set of colors $\Gamma$ is defined as follows: $\left(i^{\prime} j^{\prime}\right)<(i j)$ if either $i^{\prime}>i$ or $i^{\prime}=i$ and $j^{\prime}<j$. On the set of variables $\tilde{\Gamma}$ we define a linear order by: $x_{\gamma^{\prime}}\left(-r^{\prime}\right)<x_{\gamma}(-r)$ if either $-r^{\prime}<-r$ or $r^{\prime}=r$ and $\gamma^{\prime}<\gamma$. Since the algebra $\tilde{\mathfrak{g}}_{1}$ is commutative, we assume that variables in monomials from $\mathbb{C}[\tilde{\Gamma}]$ are sorted ascendingly from left to right.

Let $L\left(\Lambda_{k}\right), k=0, \ldots, \ell$ be a standard $\tilde{\mathfrak{g}}$-module of level 1 . We use a description of a combinatorial basis of $W=W\left(\Lambda_{k}\right)$ from [20,22]. Define an energy function $E: \Gamma \times \Gamma \rightarrow\{0,1,2\}$ by

$$
E\left(\left(i^{\prime} j^{\prime}\right),(i j)\right)=\left\{\begin{array}{ll}
0, & i^{\prime}>i, j^{\prime}<j, \\
1, & i^{\prime} \leq i, j^{\prime}<j \\
2, & i^{\prime} \leq i, j^{\prime} \geq j .
\end{array} \text { or } \quad i^{\prime}>i, j^{\prime} \geq j,\right.
$$

Define $\theta: \mathbb{Z} \rightarrow\{0,1\}$ by

$$
\theta(n)= \begin{cases}0, & n<0 \\ 1, & n \geq 0\end{cases}
$$

Then

$$
E\left(\left(i^{\prime} j^{\prime}\right),(i j)\right)=\theta\left(i-i^{\prime}\right)+\theta\left(j^{\prime}-j\right) .
$$

We say that a monomial

$$
\underline{x}=x_{i_{n} j_{n}}\left(-r_{n}\right) \cdots x_{i_{1} j_{1}}\left(-r_{1}\right) \in \mathbb{C}\left[\tilde{\Gamma}^{-}\right]
$$

satisfies difference conditions, or DC for short, if

$$
r_{t+1}-r_{t} \geq E\left(\left(i_{t+1} j_{t+1}\right),\left(i_{t} j_{t}\right)\right) .
$$

We say that $\underline{x}$ satisfies initial conditions for $L\left(\Lambda_{k}\right)$, or IC for short, if either $r_{1} \geq 2$ or $r_{1}=1$ and either $i_{1}>k$, for $1 \leq k \leq m$, or $j_{1}<k$, for $m \leq k \leq \ell$. Define

$$
\mathcal{B}_{W}=\left\{\underline{x} \in \mathbb{C}\left[\tilde{\Gamma}^{-}\right] \mid \underline{x} \text { satisfies DC and IC for } L\left(\Lambda_{k}\right)\right\} .
$$

Theorem $3.1([20,22])$. The set $\left\{\underline{x} v_{\Lambda_{k}} \mid \underline{x} \in \mathcal{B}_{W}\right\}$ is a basis of $W$. 
For a monomial $\underline{x}=x_{\gamma_{n}}\left(-r_{n}\right) \cdots x_{\gamma_{1}}\left(-r_{1}\right) \in \mathbb{C}\left[\tilde{\Gamma}^{-}\right]$, define weight and degree by

$$
w(\underline{x})=\gamma_{1}+\cdots+\gamma_{n}, \quad d(\underline{x})=r_{1}+\cdots+r_{n} .
$$

For $\alpha \in P$, set $\underline{z}^{\alpha}=z_{1}^{\left\langle\alpha, \omega_{1}\right\rangle} \cdots z_{\ell}^{\left\langle\alpha, \omega_{\ell}\right\rangle}$. The character of $W$ is the formal sum

$$
\chi_{W}\left(z_{1}, \ldots, z_{\ell}, q\right)=\sum_{\underline{x} \in \mathcal{B}_{W}} q^{d(\underline{x})} \underline{z}^{w(\underline{x})} .
$$

For a fixed $\alpha=n_{1} \alpha_{1}+\cdots+n_{\ell} \alpha_{\ell} \in P^{+}$, define $\mathcal{B}_{W}^{\alpha}=\left\{\underline{x} \in \mathcal{B}_{W} \mid w(\underline{x})=\alpha\right\}$ and $\chi_{W}^{\alpha}(q)=\sum_{\underline{x} \in \mathcal{B}_{W}^{\alpha}} q^{d(\underline{x})}$. Obviously, $\chi_{W}\left(z_{1}, \ldots, z_{\ell}, q\right)=\sum_{\alpha \in P^{+}} \chi_{W}^{\alpha}(q) \underline{z}^{\alpha}$. We sometimes use symbols $\mathcal{B}_{W}^{n_{1}, \ldots, n_{\ell}}, \chi_{W}^{n_{1}, \ldots, n_{\ell}}(q)$ instead of $\mathcal{B}_{W}^{\alpha}$ and $\chi_{W}^{\alpha}(q)$.

From (3.1) it immediately follows that $\chi_{W}^{\alpha}(q)=0$ unless $0 \leq n_{1} \leq \cdots \leq$ $n_{m} \geq \cdots \geq n_{\ell} \geq 0$.

A nondecreasing sequence of nonnegative integers $\lambda=\left(\lambda_{1}, \ldots, \lambda_{n}\right), 0 \leq$ $\lambda_{1} \leq \cdots \leq \lambda_{n}$ is called $a$ partition of length at most $n$. The sum $|\lambda|=\sum_{i} \lambda_{i}$ is called weight of $\lambda$. Denote by $\pi_{n}$ the set of partitions of length at most $n$.

For a monomial $\underline{x}=x_{\gamma_{n}}\left(-r_{n}\right) \cdots x_{\gamma_{1}}\left(-r_{1}\right) \in \mathbb{C}\left[\tilde{\Gamma}^{-}\right]$and a partition $\lambda \in$ $\pi_{n}$ define monomials

$$
\begin{aligned}
\underline{x}^{ \pm \nabla} & =x_{\gamma_{n}}\left(-r_{n} \pm(n-1)\right) \cdots x_{\gamma_{2}}\left(-r_{2} \pm 1\right) x_{\gamma_{1}}\left(-r_{1}\right), \\
\underline{x}^{ \pm} & =x_{\gamma_{n}}\left(-r_{n} \pm 1\right) \cdots x_{\gamma_{1}}\left(-r_{1} \pm 1\right), \\
\underline{x}^{ \pm r} & =x_{\gamma_{n}}\left(-r_{n} \pm r\right) \cdots x_{\gamma_{1}}\left(-r_{1} \pm r\right), \quad \text { for } r \in \mathbb{N}, \\
\underline{x}(\lambda) & =x_{\gamma_{n}}\left(-n-\lambda_{n}\right) \cdots x_{\gamma_{1}}\left(-1-\lambda_{1}\right) .
\end{aligned}
$$

We emphasize that the monomial $\underline{x}$ is assumed to be sorted ascendingly from left to right. Note that if $\underline{x}$ satisfies difference and initial conditions, then the variables in $\underline{x}$ are sorted in this way.

3.1. Character formula in the case $\omega=\omega_{1}$ or $\omega=\omega_{\ell}$. Consider the second case, $\omega=\omega_{\ell}$; the first case can be treated analogously. Fix $W=$ $W\left(\Lambda_{k}\right), 0 \leq k \leq \ell$.

The set of colors in this case is $\Gamma=\{(1 \ell), \ldots,(\ell \ell)\}$. For simplicity, we write $(i)$ and $x_{i}$ instead of $(i \ell)$ and $x_{i \ell}$, for $i=1, \ldots, \ell$. The formula (3.2) for the energy function in this case takes a simpler form:

$$
E\left(\left(i^{\prime}\right),(i)\right)=\theta\left(i-i^{\prime}\right)+1 .
$$

Set $E^{\prime}\left(\left(i^{\prime}\right),(i)\right)=E\left(\left(i^{\prime}\right),(i)\right)-1=\theta\left(i-i^{\prime}\right)$. We say that a monomial $\underline{x}=$ $x_{i_{n}}\left(-r_{n}\right) \cdots x_{i_{1}}\left(-r_{1}\right) \in \mathbb{C}\left[\tilde{\Gamma}^{-}\right]$satisfies DC' if $r_{t+1}-r_{t} \geq E^{\prime}\left(\left(i_{t+1}\right),\left(i_{t}\right)\right)$. The following lemma is obvious

Lemma 3.2. A monomial $\underline{x}$ satisfies $D C$ if and only if $\underline{x}^{+\nabla}$ satisfies $D C$ '.

Fix $0 \leq n_{1} \leq n_{2} \leq \cdots \leq n_{\ell}$ and set $\alpha=n_{1} \alpha_{1}+\cdots+n_{\ell} \alpha_{\ell}$. Set $n_{i}^{\prime}=n_{i}-n_{i-1}$, for $i=2, \ldots, \ell$, and $n_{1}^{\prime}=n_{1}$; then $\alpha=n_{1}^{\prime}(1)+\cdots+$ $n_{\ell}^{\prime}(\ell)$. Let $\underline{\lambda}=\left(\lambda^{1}, \ldots, \lambda^{\ell}\right) \in \pi_{n_{1}^{\prime}} \times \cdots \times \pi_{n_{\ell}^{\prime}}$. For $i=1, \ldots, \ell$, let us 
define $\underline{x}_{i}=x_{i}\left(-n_{i}^{\prime}-\theta(k-i)\right) x_{i}\left(-n_{i}^{\prime}+1-\theta(k-i)\right) \cdots x_{i}(-1-\theta(k-i))$. Set $\underline{x}(\underline{\lambda})=\underline{x}_{1}\left(\lambda^{1}\right) \cdots \underline{x}_{\ell}\left(\lambda^{\ell}\right)$, and reorder variables inside so that they are sorted ascendingly from left to right. Then obviously $\underline{x}(\underline{\lambda})$ satisfies DC' and IC for $L\left(\Lambda_{k}\right)$. Hence, by lemma $3.2, \underline{x}(\underline{\lambda})^{-\nabla} \in \mathcal{B}_{W}^{\alpha}$.

Conversely, let $\underline{x} \in \mathcal{B}_{W}^{\alpha}$. Set $\underline{x}^{\prime}=\underline{x}^{+\nabla}$. Factorize $\underline{x}^{\prime}=\underline{x}_{1} \cdots \underline{x}_{\ell}$ so that $\underline{x}_{i}=x_{i}\left(-r_{n_{i}^{\prime}}^{i}\right) \cdots x_{i}\left(-r_{1}^{i}\right)$ and $r_{n_{i}^{\prime}}^{i}>\cdots>r_{1}^{i}>0$ (this is possible since $\left.E^{\prime}((i),(i))=1\right)$. Define $\lambda_{t}^{i}=r_{t}^{i}-t$ for $i=1, \ldots, \ell, t=1, \ldots, n_{i}^{\prime}$. Then obviously $\lambda^{i}=\left(\lambda_{1}^{i}, \ldots, \lambda_{n_{i}^{\prime}}^{i}\right) \in \pi_{n_{i}^{\prime}}$. We have proved

THEOREM 3.3. The map

$$
\begin{aligned}
\pi_{n_{1}} \times \pi_{n_{2}-n_{1}} \cdots \times \pi_{n_{\ell}-n_{\ell-1}} & \rightarrow \mathcal{B}_{W}^{\alpha}, \\
\underline{\lambda} & \mapsto \underline{x}(\underline{\lambda})^{-\nabla}
\end{aligned}
$$

is a bijection.

Obviously

$$
\begin{aligned}
d\left(\underline{x}(\underline{\lambda})^{-\nabla}\right)= & |\lambda|+\sum_{i=1}^{\ell} \frac{n_{i}^{\prime}\left(n_{i}^{\prime}+1\right)}{2} \\
& +\frac{\left(n_{1}^{\prime}+\cdots+n_{\ell}^{\prime}\right)\left(n_{1}^{\prime}+\cdots+n_{\ell}^{\prime}-1\right)}{2}+\sum_{i=1}^{k} n_{i}^{\prime} \\
= & |\lambda|+\sum_{i=1}^{\ell} n_{i}^{2}-\sum_{i=1}^{\ell-1} n_{i} n_{i+1}+n_{k} .
\end{aligned}
$$

As a consequence, we have

Corollary 3.4. For $0 \leq n_{1} \leq n_{2} \leq \cdots \leq n_{\ell}, \alpha=n_{1} \alpha_{1}+\cdots+n_{\ell} \alpha_{\ell}$,

$$
\chi_{W\left(\Lambda_{k}\right)}^{\alpha}(q)=\frac{q^{\sum_{i=1}^{\ell} n_{i}^{2}-\sum_{i=1}^{\ell-1} n_{i} n_{i+1}+n_{k}}}{(q)_{n_{1}}(q)_{n_{2}-n_{1}} \cdots(q)_{n_{\ell}-n_{\ell-1}}},
$$

where $(q)_{n}=(1-q) \cdots\left(1-q^{n}\right)$.

REMARK 3.5. Analogous formula can be obtained in the $\omega=\omega_{1}$ case; for $n_{1} \geq n_{2} \geq \cdots \geq n_{\ell} \geq 0$ we have

$$
\chi_{W\left(\Lambda_{k}\right)}^{\alpha}(q)=\frac{q^{\sum_{i=1}^{\ell} n_{i}^{2}-\sum_{i=1}^{\ell-1} n_{i} n_{i+1}+n_{k}}}{(q)_{n_{\ell}}(q)_{n_{\ell-1}-n_{\ell}} \cdots(q)_{n_{1}-n_{2}}},
$$

3.2. Character formula in the case $\omega=\omega_{m}, 1<m<\ell$. Define Lie subalgebras $\mathfrak{g}^{\prime}=\left\langle x_{\alpha_{i}}, x_{-\alpha_{i}}, \alpha_{i} \mid i=1, \ldots, m\right\rangle$ and $\mathfrak{g}^{\prime \prime}=\left\langle x_{\alpha_{i}}, x_{-\alpha_{i}}, \alpha_{i}\right| i=$ $m, \ldots, \ell\rangle$ of types $A_{m}$ and $A_{\ell-m+1}$, respectively. We regard $\alpha_{1}, \ldots, \alpha_{m}$ and $\alpha_{m}, \ldots, \alpha_{\ell}$ as root bases, and $\omega_{1}, \ldots, \omega_{m}$ and $\omega_{m}, \ldots, \omega_{\ell}$ as fundamental weights for these subalgebras. Also, we regard $\Lambda_{0}, \Lambda_{1}, \ldots, \Lambda_{m}$ and $\Lambda_{0}, \Lambda_{m}, \ldots, \Lambda_{\ell}$ as fundamental weights for the corresponding affine Lie 
algebras. It will be clear from the context when the symbols $L\left(\Lambda_{k}\right), W\left(\Lambda_{k}\right)$, $k=0, \ldots, \ell$ denote the standard module and the corresponding FeiginStoyanovsky'y type subspace for $\mathfrak{g}$, when for $\mathfrak{g}^{\prime}$ and when for $\mathfrak{g}^{\prime \prime}$.

The set of colors $\Gamma$ is parameterized by two sets of indices - the set of row-indices $\Gamma_{1}=\{1, \ldots, m\}$ and the set of column-indices $\Gamma_{2}=\{m, \ldots, \ell\}$. We regard these two sets as sets of colors for $\mathfrak{g}^{\prime}$ and $\mathfrak{g}^{\prime \prime}$, for the choice of minuscule weight $\omega=\omega_{m}$ in both cases. Energy functions for $\Gamma_{1}$ and $\Gamma_{2}$ are

$$
E_{1}\left(i^{\prime}, i\right)=\theta\left(i-i^{\prime}\right)+1, \quad E_{2}\left(j^{\prime}, j\right)=\theta\left(j^{\prime}-j\right)+1,
$$

for $i, i^{\prime} \in \Gamma_{1}$ and $j, j^{\prime} \in \Gamma_{2}$ (see (3.6)). By (3.3), we have

$$
E\left(\left(i^{\prime} j^{\prime}\right),(i j)\right)=E_{1}\left(i^{\prime}, i\right)+E_{2}\left(j^{\prime}, j\right)-2 .
$$

We consider the case $W=W\left(\Lambda_{0}\right)$ in detail, the other cases work in the analogous manner.

A path is a finite sequence of colors $\mathbf{p}=\left(\gamma_{n}, \ldots, \gamma_{1}\right)$. The number $l(\mathbf{p})=n$ is called length of $\mathbf{p}$. The sum $w(\mathbf{p})=\gamma_{1}+\cdots+\gamma_{n}$ is called weight of $\mathbf{p}$.

To each monomial $\underline{x}=x_{\gamma_{n}}\left(-r_{n}\right) \cdots x_{\gamma_{1}}\left(-r_{1}\right) \in \mathbb{C}\left[\tilde{\Gamma}^{-}\right]$we attach its path $\mathbf{p}(\underline{x})=\left(\gamma_{n}, \ldots, \gamma_{1}\right)$. Obviously $w(\mathbf{p}(\underline{x}))=w(\underline{x})$.

Conversely, to a fixed path $\mathbf{p}=\left(\gamma_{n}, \ldots, \gamma_{1}\right)$ we attach a monomial $\underline{x}(\mathbf{p})=$ $x_{\gamma_{n}}\left(-r_{n}\right) \cdots x_{\gamma_{1}}\left(-r_{1}\right)$ such that

$$
r_{1}=1, \quad r_{t}=r_{t-1}+E\left(\gamma_{t}, \gamma_{t-1}\right) \text { for } t=2, \ldots, n \text {. }
$$

This is the "minimal" monomial of path $\mathbf{p}$ that satisfies difference and initial conditions for $L\left(\Lambda_{0}\right)$. By this we mean that if $\lambda=\left(\lambda_{1}, \ldots, \lambda_{n}\right) \in \pi_{n}$ is a partition of length at most $n$, then the monomial $(\underline{x}(\mathbf{p}))(\lambda)=x_{\gamma_{n}}\left(-r_{n}-\right.$ $\left.\lambda_{n}\right) \cdots x_{\gamma_{1}}\left(-r_{1}-\lambda_{1}\right)$ also satisfies difference and initial conditions, and all monomials of path $\mathbf{p}$ that satisfy difference and initial conditions can be obtained in this way.

Fix $0 \leq n_{1} \leq \cdots \leq n_{m} \geq \cdots \geq n_{\ell} \geq 0$ and set $\alpha=n_{1} \alpha_{1}+\cdots+n_{\ell} \alpha_{\ell}$. The argument from the preceding paragraph shows that

$$
\chi_{W\left(\Lambda_{0}\right)}^{\alpha}(q)=\frac{1}{(q)_{n_{m}}} \sum_{\mathbf{p}, w(\mathbf{p})=\alpha} q^{d(\underline{x}(\mathbf{p}))},
$$

since $l(\mathbf{p})=n_{m}$ for a path $\mathbf{p}$ of weight $\alpha$.

Fix a path $\mathbf{p}=\left(\left(i_{n_{m}} j_{n_{m}}\right), \ldots,\left(i_{1} j_{1}\right)\right)$ in $\Gamma$ of weight $\alpha$. Then, by (3.1),

(3.12) $n_{1}=\#\left\{t \mid i_{t}=1\right\}, \quad n_{s}-n_{s-1}=\#\left\{t \mid i_{t}=s\right\}$, for $s=2, \ldots, m$,

(3.13) $n_{\ell}=\#\left\{t \mid j_{t}=\ell\right\}, \quad n_{s}-n_{s+1}=\#\left\{t \mid j_{t}=s\right\}$, for $s=m, \ldots, \ell-1$.

Denote by $\mathbf{p}_{1}=\left(i_{n_{m}}, \ldots, i_{1}\right)$ and $\mathbf{p}_{2}=\left(j_{n_{m}}, \ldots, j_{1}\right)$ the corresponding paths in $\Gamma_{1}$ and $\Gamma_{2}$. Weights of $\mathbf{p}_{1}$ and $\mathbf{p}_{2}$ are $\alpha^{\prime}=n_{1} \alpha_{1}+\cdots+n_{m} \alpha_{m}$ and $\alpha^{\prime \prime}=n_{m} \alpha_{m}+\cdots+n_{\ell} \alpha_{\ell}$. Conversely, if $\mathbf{p}_{1}$ and $\mathbf{p}_{2}$ are paths in $\Gamma_{1}$ and $\Gamma_{2}$ of weights $\alpha^{\prime}$ and $\alpha^{\prime \prime}$, respectively, then the corresponding path in $\Gamma$ will be of weight $\alpha$ (cf. (3.1), (3.12), (3.13)). 
Let $\underline{x}(\mathbf{p})=x_{i_{n_{m}} j_{n_{m}}}\left(-r_{n_{m}}\right) \cdots x_{i_{1} j_{1}}\left(-r_{1}\right), \underline{x}\left(\mathbf{p}_{1}\right)=x_{i_{n_{m}}}\left(-r_{n_{m}}^{\prime}\right) \cdots$ $x_{i_{1}}\left(-r_{1}^{\prime}\right), \underline{x}\left(\mathbf{p}_{2}\right)=x_{j_{n_{m}}}\left(-r_{n_{m}}^{\prime \prime}\right) \cdots x_{j_{1}}\left(-r_{1}^{\prime \prime}\right)$ be like in (3.10). Then, by (3.3), (3.9) and (3.10), we have

$$
r_{1}=r_{1}^{\prime}=r_{1}^{\prime \prime}=1
$$

and

$$
\left\{\begin{aligned}
r_{t} & =r_{t-1}+\theta\left(i_{t-1}-i_{t}\right)+\theta\left(j_{t}-j_{t-1}\right) \\
r_{t}^{\prime} & =r_{t-1}^{\prime}+\theta\left(i_{t-1}-i_{t}\right)+1 \\
r_{t}^{\prime \prime} & =r_{t-1}^{\prime}+\theta\left(j_{t}-j_{t-1}\right)+1
\end{aligned}\right.
$$

for $t=2, \ldots, n_{m}$. By induction, from this we obtain

$$
r_{t}=r_{t}^{\prime}+r_{t}^{\prime \prime}-2 t+1, \quad \text { for } t=1, \ldots, n_{m} .
$$

This implies

$$
d(\underline{x}(\mathbf{p}))=d\left(\underline{x}\left(\mathbf{p}_{1}\right)\right)+d\left(\underline{x}\left(\mathbf{p}_{2}\right)\right)-n_{m}^{2} .
$$

Consequently

$$
\begin{aligned}
\sum_{\mathbf{p}, w(\mathbf{p})=\alpha} q^{d(\underline{x}(\mathbf{p}))} & =\sum_{\substack{\mathbf{p}_{1}, w\left(\mathbf{p}_{1}\right)=\alpha^{\prime} \\
\mathbf{p}_{2}, w\left(\mathbf{p}_{2}\right)=\alpha^{\prime \prime}}} q^{d\left(\underline{x}\left(\mathbf{p}_{1}\right)\right)+d\left(\underline{x}\left(\mathbf{p}_{2}\right)\right)-n_{m}^{2}} \\
& =\frac{1}{q^{n_{m}^{2}}}\left(\sum_{\mathbf{p}_{1}, w\left(\mathbf{p}_{1}\right)=\alpha^{\prime}} q^{d\left(\underline{x}\left(\mathbf{p}_{1}\right)\right)}\right)\left(\sum_{\mathbf{p}_{2}, w\left(\mathbf{p}_{2}\right)=\alpha^{\prime \prime}} q^{d\left(\underline{x}\left(\mathbf{p}_{2}\right)\right)}\right) .
\end{aligned}
$$

Thus, from (3.11) we obtain

$$
\chi_{W}^{\alpha}(q)=\frac{(q)_{n_{m}}}{q^{n_{m}^{2}}} \chi_{\mathfrak{g}^{\prime}, W\left(\Lambda_{0}\right)}^{\alpha^{\prime}}(q) \chi_{\mathfrak{g}^{\prime \prime}, W\left(\Lambda_{0}\right)}^{\alpha^{\prime \prime}}(q),
$$

where $\chi_{\mathfrak{g}^{\prime}, W\left(\Lambda_{0}\right)}^{\alpha^{\prime}}(q)$ and $\chi_{\mathfrak{g}^{\prime \prime}, W\left(\Lambda_{0}\right)}^{\alpha^{\prime \prime}}(q)$ are character formulas for Feigin-Stoyanovsky's type subspaces $W\left(\Lambda_{0}\right)$ for $\mathfrak{g}^{\prime}$ and $\mathfrak{g}^{\prime \prime}$, respectively. Formulas (3.7) and (3.8) give

$$
\chi_{W\left(\Lambda_{0}\right)}^{n_{1}, \ldots, n_{\ell}}(q)=\frac{q^{\sum_{i=1}^{\ell} n_{i}^{2}-\sum_{i=1}^{\ell-1} n_{i} n_{i+1}}(q)_{n_{m}}}{(q)_{n_{1}}(q)_{n_{2}-n_{1}} \cdots(q)_{n_{m}-n_{m-1}}(q)_{n_{m}-n_{m+1}} \cdots(q)_{n_{\ell-1}-n_{\ell}}(q)_{n_{\ell}}} .
$$

In other cases, when $1 \leq k \leq \ell$, the reasoning is similar, one only needs to slightly modify definitions of $\underline{x}(\mathbf{p}), \underline{x}\left(\mathbf{p}_{1}\right)$ and $\underline{x}\left(\mathbf{p}_{2}\right)$ by setting

$$
r_{1}=r_{1}^{\prime}=1+\theta\left(k-i_{1}\right), r_{1}^{\prime \prime}=1 \text {, }
$$

if $1 \leq k \leq m$, or

$$
r_{1}=r_{1}^{\prime \prime}=1+\theta\left(j_{1}-k\right), r_{1}^{\prime}=1,
$$

if $m<k \leq \ell$. In the first case, $\underline{x}(\mathbf{p}), \underline{x}\left(\mathbf{p}_{1}\right)$ and $\underline{x}\left(\mathbf{p}_{2}\right)$ are the smallest monomials of paths $\mathbf{p}, \mathbf{p}_{1}, \mathbf{p}_{2}$, that satisfy difference and initial conditions for 
$L\left(\Lambda_{k}\right), L\left(\Lambda_{k}\right)$ and $L\left(\Lambda_{0}\right)$, respectively. In the second case, these are the smallest monomials of paths $\mathbf{p}, \mathbf{p}_{1}, \mathbf{p}_{2}$ that satisfy difference and initial conditions for $L\left(\Lambda_{k}\right), L\left(\Lambda_{0}\right)$ and $L\left(\Lambda_{k}\right)$, respectively. Like in (3.19), for $1 \leq k \leq m$ we have

$$
\chi_{W\left(\Lambda_{k}\right)}^{\alpha}(q)=\frac{(q)_{n_{m}}}{q^{n_{m}^{2}}} \chi_{\mathfrak{g}^{\prime}, W\left(\Lambda_{k}\right)}^{\alpha^{\prime}}(q) \chi_{\mathfrak{g}^{\prime \prime}, W\left(\Lambda_{0}\right)}^{\alpha^{\prime \prime}}(q),
$$

while for $m<k \leq \ell$ we have

$$
\chi_{W\left(\Lambda_{k}\right)}^{\alpha}(q)=\frac{(q)_{n_{m}}}{q^{n_{m}^{2}}} \chi_{\mathfrak{g}^{\prime}, W\left(\Lambda_{0}\right)}^{\alpha^{\prime}}(q) \chi_{\mathfrak{g}^{\prime \prime}, W\left(\Lambda_{k}\right)}^{\alpha^{\prime \prime}}(q) .
$$

$$
\chi_{W\left(\Lambda_{k}\right)}^{n_{1}, \ldots, n_{\ell}}(q)=\frac{q^{\sum_{i=1}^{\ell} n_{i}^{2}-\sum_{i=1}^{\ell-1} n_{i} n_{i+1}+n_{k}}(q)_{n_{m}}}{(q)_{n_{1}}(q)_{n_{2}-n_{1}} \cdots(q)_{n_{m}-n_{m-1}}(q)_{n_{m}-n_{m+1}} \cdots(q)_{n_{\ell-1}-n_{\ell}}(q)_{n_{\ell}}} .
$$

3.3. Recurrence relations. We say that a monomial

$$
\underline{x}=x_{i_{n} j_{n}}\left(-r_{n}\right) \cdots x_{i_{1} j_{1}}\left(-r_{1}\right) \in \mathbb{C}\left[\tilde{\Gamma}^{-}\right]
$$

satisfies $\mathrm{IC}_{i j}$ if either $r_{1} \geq 2$ or $r_{1}=1$ and $i_{1} \geq i, j_{1} \leq j$. We say that a monomial $\underline{x}$ satisfies $\mathrm{IC}_{0}$ if $r_{1} \geq 2$. Denote by

$$
\begin{aligned}
\mathcal{B}_{i j} & =\left\{\underline{x} \in \mathbb{C}\left[\tilde{\Gamma}^{-}\right] \mid \underline{x} \text { satisfies DC and } \mathrm{IC}_{i j}\right\}, \\
\mathcal{B}_{0} & =\left\{\underline{x} \in \mathbb{C}\left[\tilde{\Gamma}^{-}\right] \mid \underline{x} \text { satisfies DC and } \mathrm{IC}_{0}\right\} .
\end{aligned}
$$

Note that

$$
\left\{\begin{array}{l}
\mathcal{B}_{W\left(\Lambda_{i}\right)}=\mathcal{B}_{i+1, \ell}, \text { for } i=1, \ldots, m-1, \\
\mathcal{B}_{W\left(\Lambda_{j}\right)}=\mathcal{B}_{1, j-1}, \text { for } j=m+1, \ldots, \ell \\
\mathcal{B}_{W\left(\Lambda_{0}\right)}=\mathcal{B}_{1, \ell}, \quad \mathcal{B}_{W\left(\Lambda_{m}\right)}=\mathcal{B}_{0}
\end{array}\right.
$$

The following lemma is a direct consequence of difference and initial conditions:

Lemma 3.7. (i) Let $\underline{x} \in \mathcal{B}_{i j} ;$ factorize $\underline{x}=\underline{x}_{2} \underline{x}_{1}$ so that $\underline{x}_{1}$ contains all elements of degree -1 and $\underline{x}_{2}$ contains elements of lower degree. Let $\underline{x}_{1}=$ $x_{i_{n} j_{n}}(-1) \cdots x_{i_{1} j_{1}}(-1)$. Then $i \leq i_{1}<\cdots<i_{n} \leq m \leq j_{n}<\cdots<j_{1} \leq j$.

(ii) $\quad \underline{x} \in \mathcal{B}_{0}$ if and only if $\underline{x}^{+} \in \mathcal{B}_{1 \ell}$.

For $\alpha \in Q$, define $\mathcal{B}_{i j}^{\alpha}, \mathcal{B}_{0}^{\alpha}, \chi_{i j}^{\alpha}(q)$ and $\chi_{0}^{\alpha}(q)$ like we did before. 
Proposition 3.8. Let $\alpha=n_{1} \alpha_{1}+\cdots+n_{\ell} \alpha_{\ell}$, where $0 \leq n_{1} \leq \cdots \leq$ $n_{m} \geq \cdots \geq n_{\ell} \geq 0$. Then

$$
\begin{aligned}
\chi_{0}^{\alpha}(q)= & q^{n_{m}} \chi_{1 \ell}^{\alpha}(q), \\
\chi_{i j}^{\alpha}(q)= & \chi_{i+1, j}^{\alpha}(q)+\chi_{i, j-1}^{\alpha}(q)-\chi_{i+1, j-1}^{\alpha}(q) \\
& +q \chi_{i+1, j-1}^{\alpha-(i j)}(q)-q^{n_{m}} \chi_{1, \ell}^{\alpha-(i j)}(q) \\
& +q^{n_{m}}\left(\chi_{1, j-1}^{\alpha-(i j)}(q)-\chi_{i+1, \ell}^{\alpha-(i j)}(q)-\chi_{i+1, j-1}^{\alpha-(i j)}(q)\right), \\
& \text { for } i, j \neq m, \\
\chi_{i m}^{\alpha}(q)= & \chi_{i+1, m}^{\alpha}(q)+q \chi_{i+1, m}^{\alpha-(i m)}(q), \\
\chi_{m j}^{\alpha}(q)= & \chi_{m, j-1}^{\alpha}(q)+q \chi_{m, j-1}^{\alpha-(m j)}(q), \\
\chi_{m m}^{\alpha}(q)= & \chi_{0}^{\alpha}(q)+q^{n_{m}} \chi_{0}^{\alpha-(m m)}(q) .
\end{aligned}
$$

Proof. To prove the first relation note that if $w(\underline{x})=\alpha$ then $d\left(\underline{x}^{-}\right)=$ $n_{m}+d(\underline{x})$. The relation now follows from Lemma 3.7.

We also prove the second relation; the others are proved in a similar manner. Let $\underline{x}=x_{i_{n_{m}} j_{n_{m}}}\left(-r_{n_{m}}\right) \cdots x_{i_{1} j_{1}}\left(-r_{1}\right) \in \mathbb{C}\left[\tilde{\Gamma}^{-}\right]$. If $r_{1} \geq 2$ or $r_{1}=1$ and $\left(i_{1}, j_{1}\right) \neq(i, j)$ then $\underline{x} \in \mathcal{B}_{i j}^{\alpha}$ if and only if $\underline{x} \in \mathcal{B}_{i+1, j}^{\alpha} \cup \mathcal{B}_{i, j-1}^{\alpha}$. Note also that $\mathcal{B}_{i+1, j}^{\alpha} \cap \mathcal{B}_{i, j-1}^{\alpha}=\mathcal{B}_{i+1, j-1}^{\alpha}$. This gives the first row on the right hand side of (3.24).

Assume $r_{1}=1$ and $\left(i_{1}, j_{1}\right)=(i, j)$. Set $\underline{x}_{2}=x_{i_{n_{m}} j_{n_{m}}}\left(-r_{n_{m}}\right) \cdots x_{i_{2} j_{2}}\left(-r_{2}\right)$. If $r_{2}=1$, then, by Lemma 3.7, $\underline{x} \in \mathcal{B}_{i, j}^{\alpha}$ if and only if $\underline{x}_{2} \in \mathcal{B}_{i+1, j-1}^{\alpha-(i j)} \backslash \mathcal{B}_{0}^{\alpha-(i j)}$. Together with (3.23), this gives the second row on the right hand side of (3.24).

If $r_{2} \geq 2$, then, by difference conditions, $\underline{x} \in \mathcal{B}_{i, j}^{\alpha}$ if and only if $r_{2} \geq 3$ or $r_{2}=2$ and $i_{2}>i$ or $j_{2}<j$. This is equivalent to saying that $\underline{x}_{2}^{+} \in \mathcal{B}_{1, j-1}^{\alpha-(i j)} \cup \mathcal{B}_{i+1, \ell}^{\alpha-(i j)}$. Note also that $\mathcal{B}_{1, j-1}^{\alpha-(i j)} \cap \mathcal{B}_{i+1, \ell}^{\alpha-(i j)}=\mathcal{B}_{i+1, j-1}^{\alpha-(i j)}$. This gives the last row on the right hand side of (3.24).

Theorem 3.9. For $\omega=\omega_{1}$ or $\omega=\omega_{\ell}$, the solution of the system of recursions (3.23)-(3.27) is given by formulas (3.7) and (3.8). For $\omega=\omega_{m}$, $1<m<\ell$, the solution of (3.23)-(3.27) is given by

$$
\chi_{i j}^{\alpha}(q)=\frac{q^{\sum_{t=1}^{\ell} n_{t}^{2}-\sum_{t=1}^{\ell-1} n_{t} n_{t+1}}\left(q^{n_{i-1}+n_{j+1}}+q^{n_{m}} \frac{\left(1-q^{n_{i-1}}\right)\left(1-q^{n_{j+1}}\right)}{1-q^{n_{m}}}\right)(q)_{n_{m}}}{(q)_{n_{1}}(q)_{n_{2}-n_{1}} \cdots(q)_{n_{m}-n_{m-1}}(q)_{n_{m}-n_{m+1}} \cdots(q)_{n_{\ell-1}-n_{\ell}}(q)_{n_{\ell}}},
$$

where we set $n_{0}=n_{\ell+1}=0$.

Proof. For $\omega=\omega_{1}$ or $\omega=\omega_{\ell}$, the claim follows from (3.22). Let $\omega=\omega_{m}$, $1<m<\ell$. If $i=1$ or $j=\ell$, formula (3.28) is exactly the character formula for the corresponding Feigin-Stoyanovsky's type subspace (see (3.22)). 
If $i>1$ and $j<\ell$, we find $\chi_{i j}^{\alpha}(q)$ similarly to the way we have computed characters in the previous subsection. We use the same notation as in subsection 3.2. For a path $\mathbf{p}$, define monomials $\underline{x}(\mathbf{p}), \underline{x}\left(\mathbf{p}_{1}\right)$ and $\underline{x}\left(\mathbf{p}_{2}\right)$ by (3.15), but this time, instead of (3.14), we set

$$
\begin{aligned}
& r_{1}=1+\max \left\{\theta\left(i-1-i_{1}\right), \theta\left(j_{1}-j-1\right)\right\}, \\
& r_{1}^{\prime}=1+\theta\left(i-1-i_{1}\right), \quad r_{1}^{\prime \prime}=1+\theta\left(j_{1}-j-1\right) .
\end{aligned}
$$

The difference from the previous case is that now formulas (3.16) and (3.17) fail for a path $\mathbf{p}$ that starts with a color $\left(i_{1} j_{1}\right)$ such that $i_{1}<i$ and $j_{1}>j$. For such path, we have $r_{1}=r_{1}^{\prime}=r_{1}^{\prime \prime}=2$, so (3.16) does not hold for $t=1$. This means that formula $(3.17)$ calculates $d(\underline{x}(\mathbf{p}))$ as if $r_{1}=3$ instead of $r_{1}=2$, and the difference between the calculated and the actual degree for monomials of such path is equal to $l(\mathbf{p})=n_{m}$.

Although we cannot use (3.19) to calculate $\chi_{i j}^{\alpha}(q)$, we can "repair" the wrong character formula obtained from (3.19) by recalculating degrees of monomials that start with a color $\left(i_{1} j_{1}\right)$ such that $i_{1}<i, j_{1}>j$. Let

$$
\begin{aligned}
\mathfrak{C}_{i j}^{\alpha} & =\left\{x_{i_{n_{m}} j_{n_{m}}}\left(-r_{n_{m}}\right) \cdots x_{i_{1} j_{1}}\left(-r_{1}\right) \in \mathcal{B}_{i j}^{\alpha} \mid i_{1}<i, j_{1}>j\right\}, \\
\mathfrak{D}_{i j}^{\alpha} & =\left\{x_{i_{n_{m}} j_{n_{m}}}\left(-r_{n_{m}}\right) \cdots x_{i_{1} j_{1}}\left(-r_{1}\right) \in \mathcal{B}_{1 \ell}^{\alpha} \mid r_{1}=1, i_{1}<i, j_{1}>j\right\} .
\end{aligned}
$$

Denote by $\chi_{\mathfrak{C}_{i j}}^{\alpha}(q)$ and $\chi_{\mathfrak{D}_{i j}}^{\alpha}(q)$ the corresponding graded cardinalities. By observations above, we have

$$
\chi_{i j}^{\alpha}(q)=\frac{(q)_{n_{m}}}{q^{n_{m}^{2}}} \chi_{\mathfrak{g}^{\prime}, W\left(\Lambda_{i-1}\right)}^{\alpha^{\prime}}(q) \chi_{\mathfrak{g}^{\prime \prime}, W\left(\Lambda_{j+1}\right)}^{\alpha^{\prime \prime}}(q)-\left(1-q^{n_{m}}\right) \chi_{\mathfrak{C}_{i j}}^{\alpha}(q) .
$$

Since $\underline{x} \in \mathfrak{C}_{i j}^{\alpha}$ if and only if $\underline{x}^{+r} \in \mathfrak{D}_{i j}^{\alpha}$, for some $r \in \mathbb{N}$, we have

$$
\chi_{\mathfrak{\mathfrak { C }}_{i j}}^{\alpha}(q)=\frac{q^{n_{m}}}{1-q^{n_{m}}} \chi_{\mathfrak{D}_{i j}}^{\alpha}(q) .
$$

Furthermore, since $\mathfrak{D}_{i j}^{\alpha}=\mathcal{B}_{1 \ell}^{\alpha} \backslash\left(\mathcal{B}_{1 j}^{\alpha} \cup \mathcal{B}_{i \ell}^{\alpha}\right)$ and $\mathcal{B}_{1 j}^{\alpha} \cap \mathcal{B}_{i \ell}^{\alpha}=\mathcal{B}_{i j}^{\alpha}$, we have

$$
\chi_{\mathfrak{D}_{i j}}^{\alpha}(q)=\chi_{1 \ell}^{\alpha}(q)-\chi_{i \ell}^{\alpha}(q)-\chi_{1 j}^{\alpha}(q)+\chi_{i j}^{\alpha}(q) .
$$

Consequently

$$
\begin{aligned}
\chi_{i j}^{\alpha}(q)= & \frac{(q)_{n_{m}}}{q^{n_{m}^{2}}} \chi_{\mathfrak{g}^{\prime}, W\left(\Lambda_{i-1}\right)}^{\alpha^{\prime}}(q) \chi_{\mathfrak{g}^{\prime \prime}, W\left(\Lambda_{j+1}\right)}^{\alpha^{\prime \prime}}(q) \\
& -q^{n_{m}}\left(\chi_{1 \ell}^{\alpha}(q)-\chi_{i \ell}^{\alpha}(q)-\chi_{1 j}^{\alpha}(q)+\chi_{i j}^{\alpha}(q)\right) .
\end{aligned}
$$

Formula (3.28) now follows from (3.7) and (3.8), and Theorem 3.6.

\section{The Case $D_{4}$}

4.1. Character formula for $W\left(\Lambda_{0}\right)$. Let $\mathfrak{g}$ be a simple finite-dimensional Lie algebra of type $D_{\ell}$. The minuscule fundamental weights are $\omega_{1}, \omega_{\ell-1}, \omega_{\ell}$. 
Fix a minuscule weight $\omega=\omega_{1}$. The set of colors is $\Gamma=\{\underline{2}, \ldots, \underline{\ell}, \ell, \ldots, 2\}$, where

$$
\left\{\begin{aligned}
\underline{2} & =\alpha_{1} \\
& \vdots \\
\underline{\ell} & =\alpha_{1}+\cdots+\alpha_{\ell-1} \\
\ell & =\alpha_{1}+\cdots+\alpha_{\ell-2}+\alpha_{\ell} \\
\ell-1 & =\alpha_{1}+\cdots+\alpha_{\ell-2}+\alpha_{\ell-1}+\alpha_{\ell} \\
\ell-2 & =\alpha_{1}+\cdots+\alpha_{\ell-3}+2 \alpha_{\ell-2}+\alpha_{\ell-1}+\alpha_{\ell} \\
\ell-3 & =\alpha_{1}+\cdots+\alpha_{\ell-4}+2 \alpha_{\ell-3}+2 \alpha_{\ell-2}+\alpha_{\ell-1}+\alpha_{\ell} \\
& \vdots \\
2 & =\alpha_{1}+2 \alpha_{2}+\cdots++2 \alpha_{\ell-2}+\alpha_{\ell-1}+\alpha_{\ell} .
\end{aligned}\right.
$$

Define an order on $\Gamma$ by setting: $2>\cdots>\ell>\underline{\ell}>\cdots>\underline{2}$. Like in the previous section, this induces the order on $\tilde{\Gamma}$, and we assume that monomials from $\mathbb{C}[\tilde{\Gamma}]$ are sorted ascendingly from left to right.

Let $L\left(\Lambda_{k}\right), k \in\{0,1, \ell-1, \ell\}$, be a standard $\tilde{\mathfrak{g}}$-module of level 1 , and set $W=W\left(\Lambda_{k}\right)$. Define an energy function $E: \Gamma \times \Gamma \rightarrow\{0,1,2\}$ by

$$
E\left(\gamma^{\prime}, \gamma\right)= \begin{cases}0, & \left(\gamma^{\prime}, \gamma\right)=(\underline{2}, 2), \\ 1, & \gamma^{\prime}<\gamma,\left(\gamma^{\prime}, \gamma\right) \neq(\underline{2}, 2) \text { or }\left(\gamma^{\prime}, \gamma\right)=(\ell, \underline{\ell}), \\ 2, & \gamma^{\prime} \geq \gamma,\left(\gamma^{\prime}, \gamma\right) \neq(\ell, \underline{\ell}) .\end{cases}
$$

We say that a monomial $\underline{x}=x_{\gamma_{n}}\left(-r_{n}\right) \cdots x_{\gamma_{1}}\left(-r_{1}\right) \in \mathbb{C}\left[\tilde{\Gamma}^{-}\right]$satisfies difference conditions, or DC for short, if $r_{t+1}-r_{t} \geq E\left(\gamma_{t+1}, \gamma_{t}\right)$. We say that $\underline{x}$ satisfies initial conditions for $L\left(\Lambda_{k}\right)$, or IC for short, if either $r_{1} \geq 2$ or $r_{1}=1$ and $\gamma_{1} \in\{\underline{2}, \ldots, \underline{\ell-1}, \ell\}$, for $k=\ell-1$, or $\gamma_{1} \in\{\underline{2}, \ldots, \underline{\ell}\}$, for $k=\ell$, or $\gamma_{1} \in\{\underline{2}, \ldots, \underline{\ell}, \ell, \ldots, 2\}$, for $k=0$. As before, define the set $\mathcal{B}_{W}$ by (3.4).

THEOREM $4.1([1,20])$. The set $\left\{\underline{x} v_{\Lambda_{k}} \mid \underline{x} \in \mathcal{B}_{W}\right\}$ is a basis of $W$.

From now on we assume that the algebra $\mathfrak{g}$ is of type $D_{4} ; \Gamma=$ $\{\underline{2}, \underline{3}, \underline{4}, 4,3,2\}$. Like in the previous section, we define weight and degree of monomials, and the character $\chi_{W}\left(z_{1}, z_{2}, z_{3}, z_{4}, q\right)$ of $W$. Furthermore, for $n_{1}, n_{2}, n_{3}, n_{4} \geq 0$ set $\alpha=n_{1} \alpha_{1}+n_{2} \alpha_{2}+n_{3} \alpha_{3}+n_{4} \alpha_{4}$ and define sets $\mathcal{B}_{W}^{\alpha}$ and formal series $\chi_{W}^{\alpha}(q)$ as before.

Obviously, $\chi_{W}^{\alpha}(q)=0$ unless $\alpha$ can be written in the form

$$
\alpha=m_{2} \underline{2}+m_{\underline{3}} \underline{3}+m_{\underline{4}} \underline{4}+m_{4} 4+m_{3} 3+m_{2} 2,
$$

for some $m_{\underline{2}}, m_{\underline{3}}, m_{\underline{4}}, m_{4}, m_{3}, m_{2} \in \mathbb{Z}_{\geq 0}$. Set

$$
\underline{0}=2+\underline{2}=3+\underline{3}=4+\underline{4}=2 \alpha_{1}+2 \alpha_{2}+\alpha_{3}+\alpha_{4} ;
$$

then (4.3) is equivalent to

$$
\alpha=m_{\underline{2}} \underline{2}+m_{\underline{3}} \underline{3}+m_{\underline{4}} \underline{4}+m_{\underline{0}} \underline{0},
$$


where $m_{\underline{2}}, m_{\underline{3}}, m_{\underline{4}}, m_{\underline{0}} \in \mathbb{Z}$, such that

$$
m_{\underline{0}} \geq-\theta\left(-m_{\underline{2}}\right) m_{\underline{2}}-\theta\left(-m_{\underline{3}}\right) m_{\underline{3}}-\theta\left(-m_{\underline{4}}\right) m_{\underline{4}} .
$$

By (4.1) and (4.4) we have

$$
m_{\underline{2}}=n_{1}-n_{2}, m_{\underline{3}}=n_{2}-n_{3}-n_{4}, m_{\underline{4}}=n_{3}-n_{4}, m_{\underline{0}}=n_{4} .
$$

Therefore condition (4.6) is equivalent to

$$
\begin{cases}n_{1}-n_{2}+n_{4} \geq 0, & n_{1}-n_{2}+n_{3} \geq 0 \\ n_{2}-n_{3} \geq 0, & n_{1}-n_{3} \geq 0 \\ n_{3} \geq 0, & n_{2}-n_{4} \geq 0 \\ n_{4} \geq 0, & n_{1}-n_{4} \geq 0\end{cases}
$$

We first consider the case $W=W\left(\Lambda_{0}\right)$. The other cases will be considered in the next subsection.

Set $\Gamma^{\prime}=\{\underline{2}, \underline{4}, 4,2\}, \Gamma^{\prime \prime}=\{\underline{3}, 3\}$. Define

$$
\begin{aligned}
\mathcal{B}_{\Gamma^{\prime}} & =\left\{x_{\gamma_{n}}\left(-r_{n}\right) \cdots x_{\gamma_{1}}\left(-r_{1}\right) \in \mathcal{B}_{W\left(\Lambda_{0}\right)} \mid \gamma_{i} \in \Gamma^{\prime}, i=1, \ldots, n\right\}, \\
\mathcal{B}_{\Gamma^{\prime \prime}} & =\left\{x_{\gamma_{n}}\left(-r_{n}\right) \cdots x_{\gamma_{1}}\left(-r_{1}\right) \in \mathcal{B}_{W\left(\Lambda_{0}\right)} \mid \gamma_{i} \in \Gamma^{\prime \prime}, i=1, \ldots, n\right\},
\end{aligned}
$$

$\mathcal{B}_{\Gamma^{\prime}}^{\alpha}=\mathcal{B}_{\Gamma^{\prime}} \cap \mathcal{B}_{W\left(\Lambda_{0}\right)}^{\alpha}$ and $\mathcal{B}_{\Gamma^{\prime \prime}}^{\alpha}=\mathcal{B}_{\Gamma^{\prime \prime}} \cap \mathcal{B}_{W\left(\Lambda_{0}\right)}^{\alpha}$. Define formal series $\chi_{\Gamma^{\prime}}^{\alpha}$ and $\chi_{\Gamma^{\prime \prime}}^{\alpha}$ in the obvious way.

By setting

$$
\underline{2}=(22), \underline{4}=(23), 4=(12), 2=(13),
$$

we identify the set $\Gamma^{\prime}$ with the set of colors from the case $A_{3}, \omega=\omega_{2}$ (see Section 3.2). Since the energy functions agree with this identification, and since in both cases we have the same relations between colors:

$$
\underline{2}+2=\underline{4}+4, \quad \text { i.e. } \quad(22)+(13)=(23)+(12),
$$

we conclude that the sets of monomials satisfying difference and initial conditions coincide. Therefore we can deduce a formula for $\chi_{1}^{n_{1}, n_{2}, n_{3}, n_{4}}(q)$ from the character formula for $W\left(\Lambda_{0}\right)$ for $A_{3}, \omega=\omega_{2}$. Let

$$
\alpha^{\prime}=n_{1} \alpha_{1}+n_{2} \alpha_{2}+n_{3} \alpha_{3}+n_{4} \alpha_{4}=m_{2} \underline{2}+m_{\underline{4}} \underline{4}+m_{4} 4+m_{2} 2,
$$

for some $m_{2}, m_{\underline{4}}, m_{4}, m_{2} \geq 0$. By (4.1), we have

$n_{1}=m_{\underline{2}}+m_{\underline{4}}+m_{4}+m_{2}, n_{2}=m_{\underline{4}}+m_{4}+2 m_{2}, n_{3}=m_{\underline{4}}+m_{2}, n_{4}=m_{4}+m_{2}$.

Note from (3.12), (3.13) and (4.9) that parameters $n_{1}, n_{2}-n_{1}, n_{2}-n_{3}, n_{3}$ from the case $A_{3}, \omega=\omega_{2}$, correspond to $m_{2}+m_{4}, m_{\underline{2}}+m_{\underline{4}}, m_{4}+m_{\underline{2}}, m_{\underline{4}}+$ $m_{2}$ from the $\Gamma^{\prime}$-case, respectively. From (4.11) we see that in the $\Gamma^{\prime}$-case these parameters are equal to $n_{4}, n_{1}-n_{4}, n_{1}-n_{3}, n_{3}$, respectively, and are independent of the particular choice of $m_{2}, m_{4}, m_{4}, m_{2}$. Moreover, $n_{2}=n_{1}+$ $\left(n_{2}-n_{1}\right)$ from the case $A_{3}, \omega=\omega_{2}$, corresponds to $n_{1}=m_{\underline{2}}+m_{\underline{4}}+m_{4}+m_{2}$ 
from the $\Gamma^{\prime}$-case. Hence, from character formula (3.20) for $W\left(\Lambda_{0}\right)$ for the case $A_{3}, \omega=\omega_{2}$, we get

$$
\chi_{\Gamma^{\prime}}^{\alpha^{\prime}}(q)=\frac{q^{n_{4}^{2}+n_{1}^{2}+n_{3}^{2}-n_{4} n_{1}-n_{1} n_{3}}}{(q)_{n_{3}}(q)_{n_{1}-n_{3}}(q)_{n_{1}-n_{4}}(q)_{n_{4}}} .
$$

Similarly, we identify the set $\Gamma^{\prime \prime}$ with the set of colors from the case $A_{2}, \omega=\omega_{2}$ (see Section 3.1):

$$
\underline{3}=(2), 3=(1) .
$$

Let

$$
\alpha^{\prime \prime}=n_{1} \alpha_{1}+n_{2} \alpha_{2}+n_{3} \alpha_{3}+n_{4} \alpha_{4}=m_{3} \underline{3}+m_{3} 3,
$$

for some $m_{3}, m_{3} \geq 0$. Then, by (4.1),

$$
n_{1}=m_{\underline{3}}+m_{3}, n_{2}=m_{\underline{3}}+m_{3}, n_{3}=m_{3}, n_{4}=m_{3} .
$$

From (3.13) and (4.13) we see that the parameters $n_{1}, n_{2}-n_{1}, n_{2}$ from the case $A_{2}, \omega=\omega_{2}$ correspond to $n_{4}, n_{1}-n_{4}, n_{1}$ from the $\Gamma^{\prime \prime}$-case. Hence, from character formula (3.7) for $W\left(\Lambda_{0}\right)$ for $A_{2}, \omega=\omega_{2}$, we get

$$
\chi_{\Gamma^{\prime \prime}}^{\alpha^{\prime \prime}}(q)=\frac{q^{n_{1}^{2}+n_{4}^{2}-n_{1} n_{4}}}{(q)_{n_{4}}(q)_{n_{1}-n_{4}}} .
$$

The following procedure gives us a way to obtain a character formula for $W$ from formulas (4.12) and (4.16). Set $\Gamma^{e}=\Gamma \cup\{\tilde{2}, \tilde{4}\}$ and $\tilde{\Gamma}^{e}=\tilde{\Gamma} \cup$ $\left\{x_{\gamma}(-r) \mid \gamma \in\{\tilde{2}, \tilde{4}\}, r \in \mathbb{Z}\right\}$. Define $\tilde{2}>2>3>4>\tilde{4}>\underline{4}>\underline{3}>\underline{2}$, and define the order on $\tilde{\Gamma}^{e}$ accordingly. Let $\underline{x}_{1} \in \mathcal{B}_{\Gamma^{\prime}}, \underline{x}_{2} \in \mathcal{B}_{\Gamma^{\prime \prime}}$. Denote by $\underline{x}_{3} \in \mathbb{C}\left[\tilde{\Gamma}^{e}\right]$ a monomial obtained from $\underline{x}_{1}$ by replacing every pair $x_{2}(-r) x_{2}(-r)$ with a pair $x_{\tilde{2}}(-r-1) x_{\tilde{2}}(-r)$, and every pair $x_{4}(-r-1) x_{\underline{4}}(-r)$ with a pair $x_{\tilde{4}}(-r-1) x_{\tilde{4}}(-r)$. Set $\underline{y}=\underline{x}_{3}^{+\nabla} \underline{x}_{2}^{+\nabla}$, and reorder variables so that they are sorted ascendingly from left to right. Set $\underline{z}=\underline{y}^{-\nabla}$; note that pairs $x_{\gamma}(-r-$ 1) $x_{\gamma}(-r), \gamma \in\{\tilde{2}, \tilde{4}\}$, from $\underline{x}_{3}$ correspond to pairs $x_{\gamma}\left(-r^{\prime}-1\right) x_{\gamma}\left(-r^{\prime}\right)$ from $\underline{z}$. Let $\underline{x} \in \mathbb{C}[\tilde{\Gamma}]$ be a monomial obtained from $\underline{z}$ by replacing every pair $x_{\tilde{2}}(-r-$ 1) $x_{\tilde{2}}(-r)$ inside $\underline{z}$ with a pair $x_{2}(-r) x_{2}(-r)$, and every pair $x_{\tilde{4}}(-r-1) x_{\tilde{4}}(-r)$ with a pair $x_{4}(-r-1) x_{\underline{4}}(-r)$.

Proposition 4.2. Let $\underline{x}, \underline{x}_{1}, \underline{x}_{2}$ be as above. Then $\underline{x}$ satisfies difference and initial conditions. Conversely, every monomial that satisfies difference and initial conditions can be obtained in this way.

Proof. Let $\underline{x}=x_{\gamma_{n}}\left(-r_{n}\right) \cdots x_{\gamma_{1}}\left(-r_{1}\right)$. For $t=1, \ldots, n-1$, consider factors $x_{\gamma_{t}}\left(-r_{t}\right)$ and $x_{\gamma_{t+1}}\left(-r_{t+1}\right)$. If $\gamma_{t}, \gamma_{t+1} \in \Gamma^{\prime}$ or $\gamma_{t}, \gamma_{t+1} \in \Gamma^{\prime \prime}$ then these two factors obviously satisfy difference conditions since they come from the two neighbouring factors inside $\underline{x}_{1}$ or $\underline{x}_{2}$, respectively, and the above procedure did not change the difference between their degrees. 
If $\gamma_{t} \in \Gamma^{\prime}$ and $\gamma_{t+1} \in \Gamma^{\prime \prime}$ or $\gamma_{t} \in \Gamma^{\prime \prime}$ and $\gamma_{t+1} \in \Gamma^{\prime}$, then either $r_{t+1}-r_{t}=1$ and $\gamma_{t+1}<\gamma_{t}$, or $r_{t+1}-r_{t} \geq 2$, which means that difference conditions are again satisfied.

Conversely, let $\underline{x}=x_{\gamma_{n}}\left(-r_{n}\right) \cdots x_{\gamma_{1}}\left(-r_{1}\right) \in \mathbb{C}\left[\tilde{\Gamma}^{-}\right]$be a monomial that satisfies difference and initial conditions. Let $\underline{x}_{1}$ and $\underline{x}_{2}$ be monomials obtained by the reverse procedure. The claim will follow from the following simple observations that can be proved inductively from (4.2):

(i) If $\gamma_{t}, \gamma_{t+1}, \ldots, \gamma_{t+s} \in \Gamma^{\prime}$, then $r_{t+s}-r_{t} \geq s-1$. Moreover, $r_{t+s}-r_{t}=s-1$ if and only if $s$ is odd and $\left(\gamma_{t}, \ldots, \gamma_{t+s}\right)=(2, \underline{2}, \ldots, 2, \underline{2})$.

(ii) If $\gamma_{t}, \gamma_{t+1}, \ldots, \gamma_{t+s} \in \Gamma^{\prime \prime}$, then $r_{t+s}-r_{t} \geq s+q$, where $q=\#\{0 \leq i \leq$ $\left.s-1 \mid \gamma_{t+i}<\gamma_{t+i+1}\right\}$.

First we show that $\underline{x}_{2}$ satisfies difference conditions. Let $x_{\gamma}(-r), x_{\gamma^{\prime}}\left(-r^{\prime}\right)$ be two neighbouring factors inside $\underline{x}_{2}$. Assume that $x_{\gamma_{t}}\left(-r_{t}\right)$ and $x_{\gamma_{t+s}}\left(-r_{t+s}\right)$ are the corresponding factors inside $\underline{x}$. If $s=1$, then it is obvious that $x_{\gamma}(-r)$ and $x_{\gamma^{\prime}}\left(-r^{\prime}\right)$ satisfy difference condition. If $s>1$, then $\gamma_{t+1}, \ldots, \gamma_{t+s-1} \in \Gamma^{\prime}$ and $r^{\prime}-r=r_{t+s}-r_{t}-s+1$. We need to show that either $r_{t+s}-r_{t} \geq s+1$ or $r_{t+s}-r_{t}=s$ and $\gamma>\gamma^{\prime}$. By $(i)$ and (4.2) we have:

$$
r_{t+1}-r_{t} \geq 1, r_{t+s-1}-r_{t+1} \geq s-3, r_{t+s}-r_{t+s-1} \geq 1 \text {. }
$$

Moreover, if $r_{t+s-1}-r_{t+1}=s-3$, by $(i)$ we have $\gamma_{t+s-1}=\underline{2}$ and therefore $r_{t+s}-r_{t+s-1} \geq 2$. Hence, $r_{t+s}-r_{t} \geq s$. Assume that $r_{t+s}-r_{t}=s$ (this is the case when $r^{\prime}=r+1$ ). Then (4.17) and (4.2) imply

$$
\gamma_{t}>\gamma_{t+1}
$$

Hence

$$
\gamma_{t+1} \neq 2
$$

and $r_{t+s-1}-r_{t+1}=s-2, r_{t+s}-r_{t+s-1}=1$. By (4.2), we see that

$$
\gamma_{t+s-1}>\gamma_{t+s}
$$

If $\gamma_{t+1}=\underline{2}$ then $r_{t+s-1}-r_{t+2}=s-4$, so from $(i)$ we see that $\gamma_{t+s-1}=\underline{2}$. But this is in contradiction with (4.20). If $\gamma_{t+s-1}=2$, then $r_{t+s-2}-r_{t+1}=s-4$. By $(i)$, this implies $\gamma_{t+1}=2$ which is in contradiction with (4.19). So, if $r_{t+s}-r_{t}=s$, then $\gamma_{t+1}, \gamma_{t+s-1} \in\{\underline{4}, 4\}$. By (4.18) and (4.20) we conclude that $\gamma=\gamma_{t}>\gamma_{t+s}=\gamma^{\prime}$. Therefore $x_{\gamma}(-r)$ and $x_{\gamma^{\prime}}(-r-1)$ satisfy difference conditions.

In the same way we show that $\underline{x}_{1}$ satisfies difference conditions. Let $x_{\gamma}(-r)$ and $x_{\gamma^{\prime}}\left(-r^{\prime}\right)$ be two neighbouring factors inside $\underline{x}_{1}$. Assume that $x_{\gamma_{t}}\left(-r_{t}\right)$ and $x_{\gamma_{t+s}}\left(-r_{t+s}\right)$ are the corresponding factors inside $\underline{x}$. Again, if $s=1$, the claim is obvious. Assume $s>1$. Then $\gamma_{t+1}, \ldots, \gamma_{t+s-1} \in \Gamma^{\prime \prime}$ and $r^{\prime}-r=r_{t+s}-r_{t}-s+1$. We need to show that either $r_{t+s}-r_{t} \geq s+1$ or $r_{t+s}-r_{t}=s$ and $\gamma>\gamma^{\prime}$. By $(i i)$ and (4.2) we have:

$$
r_{t+1}-r_{t} \geq 1, r_{t+s-1}-r_{t+1} \geq s+q-2, r_{t+s}-r_{t+s-1} \geq 1,
$$


where $q$ is defined in (ii). Therefore $r_{t+s}-r_{t} \geq s+q$. If $q=0$ and $r_{t+s}-r_{t}=s$, by (4.2), we must have $\gamma_{t}>\gamma_{t+1}>\cdots>\gamma_{t+s-1}>\gamma_{t+s}$. Hence $\gamma=\gamma_{t}>$ $\gamma_{t+s}=\gamma^{\prime}$ and $r^{\prime}=r+1$. We conclude that, in this case, $x_{\gamma}(-r)$ and $x_{\gamma^{\prime}}(-r-1)$ satisfy difference conditions.

By using similar arguments we can show that $\underline{x}_{1}, \underline{x}_{2} \in \mathbb{C}\left[\tilde{\Gamma}^{-}\right]$, i.e., that factors of $\underline{x}_{1}$ and $\underline{x}_{2}$ have negative degrees. Hence, $\underline{x}_{1} \in \mathcal{B}_{\Gamma^{\prime}}$ and $\underline{x}_{2} \in \mathcal{B}_{\Gamma^{\prime \prime}}$.

Let $w\left(\underline{x}_{1}\right)=n_{1}^{\prime} \alpha_{1}+n_{2}^{\prime} \alpha_{2}+n_{3}^{\prime} \alpha_{3}+n_{4}^{\prime} \alpha_{4}$ and $w\left(\underline{x}_{2}\right)=n_{1}^{\prime \prime} \alpha_{1}+n_{2}^{\prime \prime} \alpha_{2}+$ $n_{3}^{\prime \prime} \alpha_{3}+n_{4}^{\prime \prime} \alpha_{4}$. From the construction we see

$d(\underline{x})=d\left(\underline{x}_{1}\right)+d\left(\underline{x}_{2}\right)-\frac{n_{1}^{\prime}\left(n_{1}^{\prime}-1\right)}{2}-\frac{n_{1}^{\prime \prime}\left(n_{1}^{\prime \prime}-1\right)}{2}+\frac{\left(n_{1}^{\prime}+n_{1}^{\prime \prime}\right)\left(n_{1}^{\prime}+n_{1}^{\prime \prime}-1\right)}{2}$,

hence

$$
d(\underline{x})=d\left(\underline{x}_{1}\right)+d\left(\underline{x}_{2}\right)+n_{1}^{\prime} n_{1}^{\prime \prime} .
$$

Fix $n_{1}, n_{2}, n_{3}, n_{4} \geq 0$ satisfying (4.8), and set $\alpha=n_{1} \alpha_{1}+n_{2} \alpha_{2}+n_{3} \alpha_{3}+$ $n_{4} \alpha_{4}$. Define $m_{\underline{2}}, m_{\underline{3}}, m_{\underline{4}}, m_{\underline{0}}$ by (4.7). Define

$$
m^{\prime}=-\theta\left(-m_{\underline{2}}\right) m_{\underline{2}}-\theta\left(-m_{\underline{4}}\right) m_{\underline{4}}, \quad m^{\prime \prime}=-\theta\left(-m_{\underline{3}}\right) m_{\underline{3}} .
$$

Condition (4.8) is equivalent to $m_{0} \geq m^{\prime}+m^{\prime \prime}$ (cf. (4.6)). For $i=0, \ldots, m_{0}-$ $m^{\prime}-m^{\prime \prime}$, set

$$
\alpha_{i}^{\prime}=m_{\underline{2}} \underline{2}+m_{\underline{4}} \underline{4}+\left(i+m^{\prime}\right) \underline{0}, \quad \alpha_{i}^{\prime \prime}=\alpha-\alpha_{i}^{\prime} .
$$

By (4.1) and (4.4) we have

$$
\begin{aligned}
\alpha_{i}^{\prime}= & \left(n_{1}-n_{2}+n_{3}-n_{4}+2\left(i+m^{\prime}\right)\right) \alpha_{1}+\left(n_{3}-n_{4}+2\left(i+m^{\prime}\right)\right) \alpha_{2} \\
& +\left(n_{3}-n_{4}+i+m^{\prime}\right) \alpha_{3}+\left(i+m^{\prime}\right) \alpha_{4}, \\
\alpha_{i}^{\prime \prime}= & \left(n_{2}-n_{3}+n_{4}-2\left(i+m^{\prime}\right)\right) \alpha_{1}+\left(n_{2}-n_{3}+n_{4}-2\left(i+m^{\prime}\right)\right) \alpha_{2} \\
& +\left(n_{4}-i-m^{\prime}\right) \alpha_{3}+\left(n_{4}-i-m^{\prime}\right) \alpha_{4} .
\end{aligned}
$$

Then, by Proposition 4.2, (4.7) and (4.21),

$$
\chi_{W}^{\alpha}=\sum_{i=0}^{n_{4}-m^{\prime}-m^{\prime \prime}} \chi_{1}^{\alpha_{i}^{\prime}}(q) \chi_{2}^{\alpha_{i}^{\prime \prime}}(q) q^{\left(n_{1}-n_{2}+n_{3}-n_{4}+2\left(i+m^{\prime}\right)\right)\left(n_{2}-n_{3}+n_{4}-2\left(i+m^{\prime}\right)\right)} .
$$

From (4.12) and (4.16) we obtain the following character formula:

THEOREM 4.3 .

$$
\begin{aligned}
\chi_{W\left(\Lambda_{0}\right)}^{\alpha}= & \sum_{i=0}^{n_{4}-m^{\prime}-m^{\prime \prime}} q^{f^{i}(\alpha)} \frac{(q)_{n_{1}-n_{2}+n_{3}-n_{4}+2\left(i+m^{\prime}\right)}}{(q)_{n_{3}-n_{4}+i+m^{\prime}}(q)_{n_{1}-n_{2}+i+m^{\prime}}(q)_{i+m^{\prime}}} \\
& \cdot \frac{1}{(q)_{n_{1}-n_{2}+n_{3}-n_{4}+i+m^{\prime}}(q)_{n_{4}-i-m^{\prime}}(q)_{n_{2}-n_{3}-i-m^{\prime}}},
\end{aligned}
$$


where

$$
\begin{aligned}
f^{i}(\alpha)= & n_{1}^{2}+n_{2}^{2}+n_{3}^{2}+n_{4}^{2}-n_{1} n_{2}-n_{2} n_{3}-n_{3} n_{4} \\
& -\left(i+m^{\prime}\right)\left(n_{2}-n_{3}+n_{4}-i-m^{\prime}\right) .
\end{aligned}
$$

REMARK 4.4. For an algebra $\mathfrak{g}$ of type $D_{\ell}$, let $\alpha=n_{1} \alpha_{1}+\cdots+n_{\ell} \alpha_{\ell}$ be a weight that can be written as a non-negative linear combination of colors $\underline{2}, \ldots, \underline{\ell}, \ell, \ldots, 2$. Set $\underline{0}=2+\underline{2}=\cdots=\ell+\underline{\ell}=2 \alpha_{1}+\cdots+2 \alpha_{\ell-2}+\alpha_{\ell-1}+\alpha_{\ell}$. Define $m_{\underline{2}}, \ldots, m_{\underline{\ell}}, m_{\underline{0}}$ like in (4.5). Like in (4.6), we obtain the following condition on the coefficients $m_{\underline{2}}, \ldots, m_{\underline{\ell}}, m_{\underline{0}}$ :

$$
m_{\underline{0}} \geq-\theta\left(-m_{\underline{2}}\right) m_{\underline{2}}-\cdots-\theta\left(-m_{\underline{\ell}}\right) m_{\underline{\ell}} .
$$

Partition the set of colors into the sets

$$
\Gamma^{(2)}=\{\underline{2}, \underline{\ell}, \ell, 2\}, \Gamma^{(3)}=\{\underline{3}, 3\}, \ldots, \Gamma^{(\ell-1)}=\{\underline{\ell-1}, \ell-1\},
$$

and regard $\Gamma^{(2)}$ as a set of colors for the case $A_{3}, \omega=\omega_{2}$, and $\Gamma^{(3)}, \ldots, \Gamma^{(\ell-1)}$ as sets of colors for the case $A_{2}, \omega=\omega_{2}$. Set $m^{(2)}=-\theta\left(-m_{\underline{2}}\right) m_{\underline{2}}-\theta\left(-m_{\underline{\ell}}\right) m_{\underline{\ell}}$, $m^{(3)}=-\theta\left(-m_{\underline{3}}\right) m_{\underline{3}}, \ldots, m^{(\ell-1)}=-\theta\left(-m_{\underline{\ell-1}}\right) m_{\underline{\ell-1}}$, and let $m=m^{(2)}+\cdots+$ $m^{(\ell-1)}$. We can apply the same procedure as before; we obtain the following character formula:

$$
\begin{aligned}
\chi_{W\left(\Lambda_{0}\right)}^{\alpha}= & \sum_{\substack{\mathbf{i}=\left(i_{2}, \ldots, i_{\ell-1}\right) \in \mathbb{Z}_{\geq 0}^{\ell-2} \\
i_{2}+\cdots+i_{\ell-1}=n_{\ell}-m}} q^{f^{\mathbf{i}}(\alpha)} \frac{(q)_{n_{1}-n_{2}+n_{\ell-1}-n_{\ell}+2\left(i_{2}+m^{(2)}\right)}}{(q)_{n_{\ell-1}-n_{\ell}+i_{2}+m^{(2)}}(q)_{n_{1}-n_{2}+i_{2}+m^{(2)}}(q)_{i_{2}+m^{(2)}}} \\
& \cdot \frac{1}{(q)_{n_{1}-n_{2}+n_{\ell-1}-n_{\ell}+i_{2}+m^{(2)}}} \prod_{j=3}^{\ell-2} \frac{1}{(q)_{i_{j}+m^{(j)}}(q)_{n_{j-1}-n_{j}+i_{j}+m^{(j)}}} \\
& \cdot \frac{1}{(q)_{i_{\ell-1}+m^{(\ell-1)}}(q)_{n_{\ell-2}-n_{\ell-1}-n_{\ell}+i_{\ell-1}+m^{(\ell-1)}}},
\end{aligned}
$$

where

$$
\begin{aligned}
f^{\mathbf{i}}(\alpha)= & n_{1}^{2}+\cdots+n_{\ell}^{2}-n_{1} n_{2}-\cdots-n_{\ell-3} n_{\ell-2}-n_{\ell-2} n_{\ell-1}-n_{\ell-2} n_{\ell} \\
& +\sum_{j=3}^{\ell-2}\left(i_{j}+m^{(j)}\right)\left(n_{j-1}-n_{j}+i_{j}+m^{(j)}\right) \\
& +\left(i_{\ell-1}+m^{(\ell-1)}\right)\left(n_{\ell-2}-n_{\ell-1}-n_{\ell}+i_{\ell-1}+m^{(\ell-1)}\right) .
\end{aligned}
$$

4.2. Character formulas for other level 1 standard modules and recurrence relations. For $\gamma \in \Gamma$, we say that a monomial

$$
\underline{x}=x_{\gamma_{n}}\left(-r_{n}\right) \cdots x_{\gamma_{1}}\left(-r_{1}\right) \in \mathbb{C}\left[\tilde{\Gamma}^{-}\right]
$$

satisfies $\mathrm{IC}_{\gamma}$ if either $r_{1} \geq 2$ or $r_{1}=1$ and either $\gamma_{1} \leq \gamma$ if $\gamma \neq 4$, or $\gamma_{1} \in\{\underline{2}, \underline{3}, 4\}$ if $\gamma=4$. We say that a monomial $\underline{x}$ satisfies $\mathrm{IC}_{0}$ if $r_{1} \geq 2$. 
Denote by

$$
\begin{aligned}
& \mathcal{B}_{\gamma}=\left\{\underline{x} \in \mathbb{C}\left[\tilde{\Gamma}^{-}\right] \mid \underline{x} \text { satisfies } \mathrm{DC} \text { and } \mathrm{IC}_{\gamma}\right\} \\
& \mathcal{B}_{0}=\left\{\underline{x} \in \mathbb{C}\left[\tilde{\Gamma}^{-}\right] \mid \underline{x} \text { satisfies } \mathrm{DC} \text { and } \mathrm{IC}_{0}\right\}
\end{aligned}
$$

Note that

$$
\mathcal{B}_{W\left(\Lambda_{0}\right)}=\mathcal{B}_{2}, \quad \mathcal{B}_{W\left(\Lambda_{1}\right)}=\mathcal{B}_{0}, \quad \mathcal{B}_{W\left(\Lambda_{3}\right)}=\mathcal{B}_{4}, \quad \mathcal{B}_{W\left(\Lambda_{4}\right)}=\mathcal{B}_{\underline{4}} .
$$

For $n_{1}, n_{2}, n_{3}, n_{4}$ satisfying (4.8), set $\alpha=n_{1} \alpha_{1}+n_{2} \alpha_{2}+n_{3} \alpha_{3}+n_{4} \alpha_{4}$ and define $\mathcal{B}_{\gamma}^{\alpha}, \mathcal{B}_{0}^{\alpha}, \chi_{\gamma}^{\alpha}(q)$ and $\chi_{0}^{\alpha}(q)$ as before.

Proposition 4.5. Characters $\chi_{\gamma}^{\alpha}(q), \gamma \in \Gamma \cup\{0\}$, satisfy the following recurrence relations:

$$
\begin{aligned}
& \chi_{0}^{\alpha}(q)=q^{n_{1}} \chi_{2}^{\alpha}(q), \\
& \chi_{2}^{\alpha}(q)=\chi_{3}^{\alpha}(q)+q^{n_{1}} \chi_{3}^{\alpha-2}(q)+q^{2 n_{1}-2} \chi_{2}^{\alpha-2-\underline{2}}(q), \\
& \chi_{3}^{\alpha}(q)=\chi_{4}^{\alpha}(q)+\chi_{\underline{4}}^{\alpha}(q)-\chi_{\underline{3}}^{\alpha}(q)+q^{n_{1}}\left(\chi_{4}^{\alpha-3}(q)+\chi_{\underline{4}}^{\alpha-3}(q)-\chi_{\underline{3}}^{\alpha-3}(q)\right), \\
& \chi_{4}^{\alpha}(q)=\chi_{\underline{3}}^{\alpha}(q)+q^{n_{1}} \chi_{\underline{4}}^{\alpha-4}(q), \\
& \chi_{\underline{4}}^{\alpha}(q)=\chi_{\underline{3}}^{\alpha}(q)+q^{n_{1}} \chi_{4}^{\alpha-\underline{4}}(q), \\
& \chi_{\underline{3}}^{\alpha}(q)=\chi_{\underline{2}}^{\alpha}(q)+q^{n_{1}} \chi_{\underline{2}}^{\alpha-\underline{3}}(q), \\
& \chi_{\underline{2}}^{\alpha}(q)=\chi_{0}^{\alpha}(q)+q^{n_{1}} \chi_{0}^{\alpha-\underline{2}}(q) .
\end{aligned}
$$

The proof is similar to the proof in the $A_{\ell}$-case.

Set

$$
\begin{aligned}
& \mathcal{B}_{\Gamma^{\prime \prime} ; 0}=\left\{x_{\gamma_{n}}\left(-r_{n}\right) \cdots x_{\gamma_{1}}\left(-r_{1}\right) \in \mathcal{B}_{\Gamma^{\prime \prime}} \mid r_{1} \geq 2\right\}, \\
& \mathcal{B}_{\Gamma^{\prime \prime} ; \underline{3}}=\left\{x_{\gamma_{n}}\left(-r_{n}\right) \cdots x_{\gamma_{1}}\left(-r_{1}\right) \in \mathcal{B}_{\Gamma^{\prime \prime}} \mid r_{1} \geq 2 \text { or } r_{1}=1, \gamma_{1}=\underline{3}\right\}, \\
& \mathcal{B}_{\Gamma^{\prime} ; 0}=\left\{x_{\gamma_{n}}\left(-r_{n}\right) \cdots x_{\gamma_{1}}\left(-r_{1}\right) \in \mathcal{B}_{\Gamma^{\prime}} \mid r_{1} \geq 2\right\}, \\
& \mathcal{B}_{\Gamma^{\prime} ; \underline{2}}=\left\{x_{\gamma_{n}}\left(-r_{n}\right) \cdots x_{\gamma_{1}}\left(-r_{1}\right) \in \mathcal{B}_{\Gamma^{\prime}} \mid r_{1} \geq 2 \text { or } r_{1}=1, \gamma_{1}=\underline{2}\right\}, \\
& \mathcal{B}_{\Gamma^{\prime} ; 4, \underline{2}}=\left\{x_{\gamma_{n}}\left(-r_{n}\right) \cdots x_{\gamma_{1}}\left(-r_{1}\right) \in \mathcal{B}_{\Gamma^{\prime}} \mid r_{1} \geq 2 \text { or } r_{1}=1, \gamma_{1} \in\{4, \underline{2}\}\right\}, \\
& \mathcal{B}_{\Gamma^{\prime} ; \underline{4}, \underline{2}}=\left\{x_{\gamma_{n}}\left(-r_{n}\right) \cdots x_{\gamma_{1}}\left(-r_{1}\right) \in \mathcal{B}_{\Gamma^{\prime}} \mid r_{1} \geq 2 \text { or } r_{1}=1, \gamma_{1} \in\{\underline{4}, \underline{2}\}\right\}, \\
& \mathcal{B}_{\Gamma^{\prime} ; 4, \underline{4}, \underline{2}}=\left\{x_{\gamma_{n}}\left(-r_{n}\right) \cdots x_{\gamma_{1}}\left(-r_{1}\right) \in \mathcal{B}_{\Gamma^{\prime}} \mid r_{1} \geq 2 \text { or } r_{1}=1, \gamma_{1} \in\{4, \underline{4}, \underline{2}\}\right\},
\end{aligned}
$$

and define $\mathcal{B}_{\Gamma^{\prime \prime} ; 0}^{\alpha}, \mathcal{B}_{\Gamma^{\prime \prime} ; \underline{3}}^{\alpha}, \mathcal{B}_{\Gamma^{\prime} ; 0}^{\alpha}, \mathcal{B}_{\Gamma^{\prime} ; \underline{2}}^{\alpha}, \mathcal{B}_{\Gamma^{\prime} ; 4, \underline{2}}^{\alpha}, \mathcal{B}_{\Gamma^{\prime} ; \underline{4}, \underline{2}}^{\alpha}, \mathcal{B}_{\Gamma^{\prime} ; 4, \underline{4}, \underline{2}}^{\alpha}$, and $\chi_{\Gamma^{\prime \prime} ; 0}^{\alpha}, \chi_{\Gamma^{\prime \prime} ; \underline{3}}^{\alpha}$, $\chi_{\Gamma^{\prime} ; 0}^{\alpha}, \chi_{\Gamma^{\prime} ; \underline{2}}^{\alpha}, \chi_{\Gamma^{\prime} ; 4, \underline{2}}^{\alpha}, \chi_{\Gamma^{\prime} ; \underline{4}, \underline{2}}^{\alpha}, \chi_{\Gamma^{\prime} ; 4, \underline{4}, \underline{2}}^{\alpha}$ in the obvious way.

Character formulas for these sets can be obtained in the same way as we did for $\chi_{\Gamma^{\prime}}^{\alpha}$ and $\chi_{\Gamma^{\prime \prime}}^{\alpha}$ in the previous section, by using character formulas for 
cases $A_{3}$, with $\omega=\omega_{2}$, and $A_{2}$, with $\omega=\omega_{2}$, from Section 3.3. We get

$$
\begin{aligned}
\chi_{\Gamma^{\prime \prime} ; 0}^{\alpha^{\prime \prime}}= & \frac{q^{n_{1}^{2}+n_{4}^{2}-n_{1} n_{4}+n_{1}}}{(q)_{n_{4}}(q)_{n_{1}-n_{4}}} \\
\chi_{\Gamma^{\prime \prime} ; \underline{3}}^{\alpha^{\prime \prime}}= & \frac{q^{n_{1}^{2}+n_{4}^{2}-n_{1} n_{4}+n_{4}}}{(q)_{n_{4}}(q)_{n_{1}-n_{4}}}, \\
\chi_{\Gamma^{\prime} ; 0}^{\alpha^{\prime}}= & \frac{q^{n_{4}^{2}+n_{1}^{2}+n_{3}^{2}-n_{4} n_{1}-n_{1} n_{3}+n_{1}}}{(q)_{n_{3}}(q)_{n_{1}-n_{3}}(q)_{n_{1}-n_{4}}(q)_{n_{4}}}, \\
\chi_{\Gamma^{\prime} ; \underline{2}}^{\alpha^{\prime}}= & \frac{q^{n_{4}^{2}+n_{1}^{2}+n_{3}^{2}-n_{4} n_{1}-n_{1} n_{3}}}{(q)_{n_{3}}(q)_{n_{1}-n_{3}}(q)_{n_{1}-n_{4}}(q)_{n_{4}}} \\
& \cdot\left(q^{n_{4}+n_{3}}+q^{n_{1}} \frac{\left(1-q^{n_{4}}\right)\left(1-q^{n_{3}}\right)}{1-q^{n_{1}}}\right) \\
\chi_{\Gamma^{\prime} ; 4, \underline{2}}^{\alpha^{\prime}}= & \frac{q^{n_{4}^{2}+n_{1}^{2}+n_{3}^{2}-n_{4} n_{1}-n_{1} n_{3}+n_{3}}}{(q)_{n_{3}}(q)_{n_{1}-n_{3}}(q)_{n_{1}-n_{4}}(q)_{n_{4}}}, \\
\chi_{\Gamma^{\prime} ; \underline{4}, \underline{2}=}^{\alpha^{\prime}}= & \frac{q^{n_{4}^{2}+n_{1}^{2}+n_{3}^{2}-n_{4} n_{1}-n_{1} n_{3}+n_{4}}}{(q)_{n_{3}}(q)_{n_{1}-n_{3}}(q)_{n_{1}-n_{4}}(q)_{n_{4}}}, \\
\chi_{\Gamma^{\prime} ; 4, \underline{4}, \underline{2}=}^{\alpha^{\prime}}= & \chi_{\Gamma^{\prime} ; 4, \underline{2}}^{\alpha^{\prime}}+\chi_{\Gamma^{\prime} ; \underline{4}, \underline{2}}^{\alpha^{\prime}}-\chi_{\Gamma^{\prime} ;}^{\alpha^{\prime}} \\
= & \frac{q^{n_{4}^{2}+n_{1}^{2}+n_{3}^{2}-n_{4} n_{1}-n_{1} n_{3}}}{(q)_{n_{3}}(q)_{n_{1}-n_{3}}(q)_{n_{1}-n_{4}}(q)_{n_{4}}}\left(1-\frac{\left(1-q^{n_{4}}\right)\left(1-q^{n_{3}}\right)}{1-q^{n_{1}}}\right),
\end{aligned}
$$

for $\alpha^{\prime}$ and $\alpha^{\prime \prime}$ satisfying (4.10) and (4.14), respectively.

Proposition 4.6. Let $\underline{x}, \underline{x}_{1}, \underline{x}_{2}$ be like in Proposition 4.2. Then:

$$
\begin{aligned}
& \underline{x} \in \mathcal{B}_{2} \Leftrightarrow \underline{x}_{1} \in \mathcal{B}_{\Gamma^{\prime}}, \underline{x}_{2} \in \mathcal{B}_{\Gamma^{\prime \prime}}, \\
& \underline{x} \in \mathcal{B}_{3} \Leftrightarrow \underline{x}_{1} \in \mathcal{B}_{\Gamma^{\prime} ; 4,4}, \underline{2}, \underline{x}_{2} \in \mathcal{B}_{\Gamma^{\prime \prime}}, \\
& \underline{x} \in \mathcal{B}_{4} \Leftrightarrow \underline{x}_{1} \in \mathcal{B}_{\Gamma^{\prime} ; 4, \underline{2}}, \underline{x}_{2} \in \mathcal{B}_{\Gamma^{\prime \prime}}, \\
& \underline{x} \in \mathcal{B}_{4} \Leftrightarrow \underline{x}_{1} \in \mathcal{B}_{\Gamma^{\prime} ; \underline{4}, \underline{2}}, \underline{x}_{2} \in \mathcal{B}_{\Gamma^{\prime \prime} ; \underline{3}}, \\
& \underline{x} \in \mathcal{B}_{\underline{3}} \Leftrightarrow \underline{x}_{1} \in \mathcal{B}_{\Gamma^{\prime} ; \underline{2}}, \underline{x}_{2} \in \mathcal{B}_{\Gamma^{\prime \prime} ; \underline{3}}, \\
& \underline{x} \in \mathcal{B}_{\underline{2}} \Leftrightarrow \underline{x}_{1} \in \mathcal{B}_{\Gamma^{\prime} ; \underline{x}}, \underline{x}_{2} \in \mathcal{B}_{\Gamma^{\prime \prime} ; 0}, \\
& \underline{x} \in \mathcal{B}_{0} \Leftrightarrow \underline{x}_{1} \in \mathcal{B}_{\Gamma^{\prime} ; 0}, \underline{x}_{2} \in \mathcal{B}_{\Gamma^{\prime \prime} ; 0} .
\end{aligned}
$$

The proposition can be proved by arguments similar to the ones used in the proof of Proposition 4.2.

Fix $n_{1}, n_{2}, n_{3}, n_{4} \geq 0$ satisfying (4.8), and set $\alpha=n_{1} \alpha_{1}+n_{2} \alpha_{2}+n_{3} \alpha_{3}+$ $n_{4} \alpha_{4}$. Define $m_{\underline{2}}, m_{\underline{3}}, m_{\underline{4}}, m_{\underline{0}}$ by (4.7), $m^{\prime}$ and $m^{\prime \prime}$ by (4.22), and $\alpha_{i}^{\prime}$ and $\alpha_{i}^{\prime \prime}$, for $i=0, \ldots, n_{4}-m^{\prime}-m^{\prime \prime}$, by (4.23). Proposition 4.6 enables us to compute characters by using analogues of formula (4.24) and formulas (4.28)-(4.34). 


\section{Theorem 4.7.}

$$
\begin{aligned}
\chi_{\gamma}^{\alpha}= & \sum_{i=0}^{n_{4}-m^{\prime}-m^{\prime \prime}} r_{\gamma}^{i}(\alpha) q^{f^{i}(\alpha)} \frac{(q)_{n_{1}-n_{2}+n_{3}-n_{4}+2\left(i+m^{\prime}\right)}}{(q)_{n_{3}-n_{4}+i+m^{\prime}}(q)_{n_{1}-n_{2}+i+m^{\prime}}(q)_{i+m^{\prime}}} \\
& \cdot \frac{1}{(q)_{n_{1}-n_{2}+n_{3}-n_{4}+i+m^{\prime}}(q)_{n_{4}-i-m^{\prime}}(q)_{n_{2}-n_{3}-i-m^{\prime}}},
\end{aligned}
$$

where $f^{i}(\alpha)$ is defined by (4.26), and $r_{\gamma}^{i}(\alpha)$ is defined by

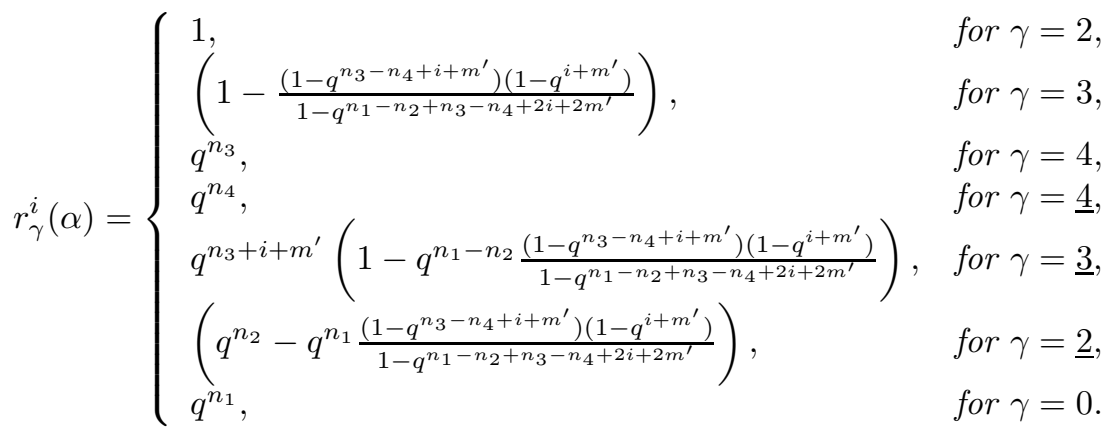

\section{REFERENCES}

[1] I. Baranović, Combinatorial bases of Feigin-Stoyanovsky's type subspaces of level 2 standard modules for $D_{4}^{(1)}$, math.QA/0903.0739.

[2] I. Baranović, in preparation.

[3] C. Calinescu, Intertwining vertex operators and certain representations of $\widehat{\mathfrak{s l}(n)}$, Commun. Contemp. Math. 10 (2008), 47-79.

[4] C. Calinescu, Principal subspaces of higher-level standard $\widehat{\mathfrak{s l}(3)}-$ modules, J. Pure Appl. Algebra 210 (2007), 559-575.

[5] C. Calinescu, J. Lepowsky and A. Milas, Vertex-algebraic structure of the principal subspaces of certain $A_{1}^{(1)}$-modules. I. Level one case, Int. J. Math. 19 (2008), 71-92.

[6] C. Calinescu, J. Lepowsky and A. Milas, Vertex-algebraic structure of the principal subspaces of certain $A_{1}^{(1)}$-modules. II. Higher level case, J. Pure Appl. Algebra 212 (2008), 1928-1950.

[7] C. Calinescu, J. Lepowsky and A. Milas, Vertex-algebraic structure of the principal subspaces of level one modules for the untwisted affine Lie algebras of types $A, D$, E, J. Algebra 323 (2010), 167-192.

[8] S. Capparelli, J. Lepowsky and A. Milas, The Rogers-Ramanujan recursion and intertwining operators, Commun. Contemp. Math. 5 (2003), 947-966.

[9] S. Capparelli, J. Lepowsky and A. Milas, The Rogers-Selberg recursions, the GordonAndrews identities and intertwining operators, Ramanujan J. 12 (2006), 379-397.

[10] B. Feigin, M. Jimbo, S. Loktev, T. Miwa and E. Mukhin, Bosonic formulas for $(k, \ell)$-admissible partitions, Ramanujan J. 7 (2003), 485-517.; Addendum to 'Bosonic formulas for $(k, \ell)$-admissible partitions', Ramanujan J. 7 (2003), 519-530.

[11] B. Feigin, M. Jimbo, T. Miwa, E. Mukhin and Y. Takeyama, Fermionic formulas for $(k, 3)$-admissible configurations, Publ. Res. Inst. Math. Sci. 40 (2004), 125-162.

[12] B. Feigin, M. Jimbo, T. Miwa, E. Mukhin and Y. Takeyama, Particle content of the $(k, 3)$-configurations, Publ. Res. Inst. Math. Sci. 40 (2004), 163-220. 
[13] A. V. Stoyanovsky and B. L. Feigin, Functional models of the representations of current algebras, and semi-infinite Schubert cells, (Russian) Funktsional. Anal. i Prilozhen. 28 (1994), 68-90, 96; translation in Funct. Anal. Appl. 28 (1994), 5572.

[14] G. Georgiev, Combinatorial constructions of modules for infinite-dimensional Lie algebras. I. Principal subspace, J. Pure Appl. Algebra 112 (1996), 247-286.

[15] M. Jerković, Recurrence relations for characters of affine Lie algebra $A_{\ell}^{(1)}$, J. Pure Appl. Algebra 213 (2009), 913-926.

[16] M. Jerković, Character formulas for Feigin-Stoyanovsky's type subspaces of standard $\mathfrak{s l}(3, \mathbb{C})^{\sim}$-modules, arXiv:1105.2927v1 [math.QA].

[17] M. Jerković, Recurrence relations for characters of affine Lie algebra $A_{l}^{(1)}, \mathrm{PhD}$ thesis, University of Zagreb, 2007.

[18] V. G. Kac, Infinite-dimensional Lie algebras. 3rd ed., Cambridge University Press, Cambridge, 1990.

[19] M. Primc, Vertex operator construction of standard modules for $A_{n}^{(1)}$, Pacific J. Math. 162 (1994), 143-187.

[20] M. Primc, Basic representations for classical affine Lie algebras, J. Algebra 228 (2000), 1-50.

[21] M. Primc, $(k, r)$-admissible configurations and intertwining operators, Contemp. Math. 442 (2007), 425-434.

[22] G. Trupčević, Combinatorial bases of Feigin-Stoyanovsky's type subspaces of level 1 standard $\tilde{\mathfrak{s l}}(\ell+1, \mathbb{C})$-modules, Commun. Algebra 38 (2010), 3913-3940.

[23] G. Trupčević, Combinatorial bases of Feigin-Stoyanovsky's type subspaces of higherlevel standard $\tilde{\mathfrak{s l}}(\ell+1, \mathbb{C})$-modules, J. Algebra 322 (2009), 3744-3774.

G. Trupčević

Department of Mathematics

University of Zagreb

Bijenička 30, 10000 Zagreb

Croatia

E-mail: gtrup@math.hr

Received: 1.2.2010. 